University of Louisville

ThinkIR: The University of Louisville's Institutional Repository

Electronic Theses and Dissertations

8-2017

\title{
Fabrication and functionalization of carbon nanocages for use in CFx batteries.
}

Nathan Smith

University of Louisville

Follow this and additional works at: https://ir.library.louisville.edu/etd

Part of the Condensed Matter Physics Commons

\section{Recommended Citation}

Smith, Nathan, "Fabrication and functionalization of carbon nanocages for use in CFx batteries." (2017). Electronic Theses and Dissertations. Paper 2805.

https://doi.org/10.18297/etd/2805

This Master's Thesis is brought to you for free and open access by ThinkIR: The University of Louisville's Institutional Repository. It has been accepted for inclusion in Electronic Theses and Dissertations by an authorized administrator of ThinkIR: The University of Louisville's Institutional Repository. This title appears here courtesy of the author, who has retained all other copyrights. For more information, please contact thinkir@louisville.edu. 


\title{
FABRICATION AND FUNCTIONALIZATION OF CARBON NANOCAGES FOR USE IN CFX BATTERIES
}

\author{
By \\ Nathan Smith \\ B. S., Georgetown College, 2015

\begin{abstract}
A Thesis
Submitted to the Faculty of the

College of Arts and Sciences of the University of Louisville

in Partial Fulfillment of the Requirements

for the Degree of
\end{abstract}

\section{Master of Science \\ in Physics} \\ Department of Physics \\ University of Louisville \\ Louisville, Kentucky
}

August 2017 



\title{
FABRICATION AND FUNCTIONALIZATION OF CARBON NANOCAGES FOR USE IN CFX BATTERIES
}

\author{
By
}

Nathan Smith

B. S., Georgetown College, 2015

A Thesis approved on

July 31,2017

by the Following Thesis Committee:

Dr. Gamini Sumanasekera (Thesis Director)

Dr. Jacek Jasinski

Dr. Ming Yu 


\section{ACKNOWLEDGEMENTS}

First, I would like to thank my thesis advisor, Dr. Gamini Sumanasekera, for his step by step guidance throughout this project. Thank you for always being available to answer my questions and encourage me. Second, I would like to thank my lab partner, Bhupendra Karki, for all his assistance throughout every phase of this project, I would not have been able to complete this project without you. I would also like to thank Dr. Jacek Jasinski for his willingness to take time to evaluate this thesis, as well as operating the TEM for me. I would like to thank Dominika Ziolkowska for her assistance in both the preparation of the precursor and the etching of the resulting samples. I would like to thank Joshua Rimmer for his machine shop help. I would like to thank Dr. Arjun Thapa for his extensive help with the battery fabrication and testing process. I would like to thank Dr. Ming Yu for her valuable input to this thesis. Finally I would like to thank my family for their endless love and support. 


\begin{abstract}
FABRICATION AND FUNCTIONALIZATION OF CARBON NANOCAGES FOR USE IN CFX BATTERIES

Nathan Smith

July 31,2017

A relatively inexpensive method for obtaining high quality lithium- $\mathrm{CF}_{\mathrm{x}}$ batteries is reported. Nickel acetate and citric acid are used to synthesize a precursor which is then annealed and subsequently etched to fabricate highly uniform $3 \mathrm{~nm}$ radius pristine CNCs. These CNCs are then functionalized with fluorine using an RF plasma system. The resulting fluorinated CNCs are then combined with a carbon binder and pressed onto a stainless steel mesh to form a $\mathrm{CF}_{\mathrm{x}}$ cathode. This cathode is then tested in a coin cell primary battery with a lithium anode. Results indicate that a specific capacity of $660 \mathrm{mAh} / \mathrm{g}$ was achieved for a sample with a calculated x value of 0.93 .
\end{abstract}




\section{TABLE OF CONTENTS}

\section{PAGE}

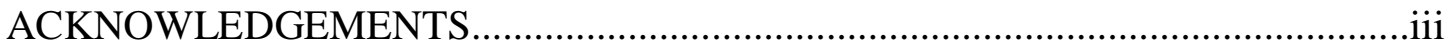

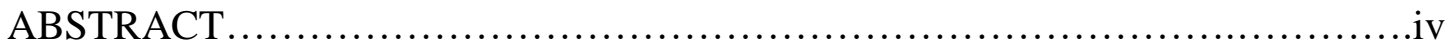

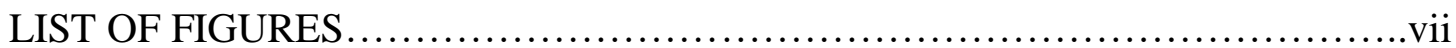

1. INTRODUCTION AND BACKGROUND .....................................

1.1 Introduction.............................................................

1.2 Electron Orbitals of Carbon............................................

1.2.1 $\mathrm{sp}$ Hybridization. .................................................

1.2.2 $\mathrm{sp}^{2}$ Hybridization................................................

$1.2 .3 \mathrm{sp}^{3}$ Hybridization................................................4

1.3 Discovery of Graphene.................................................

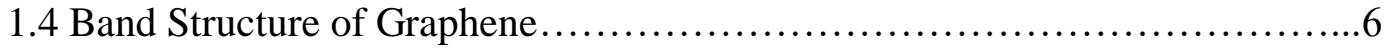

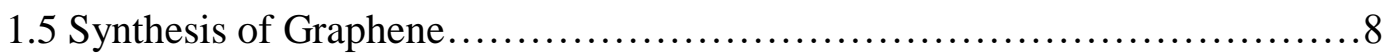

1.6 Engineering a Band Gap in Graphene..................................9

1.6.1 Substrate Induced Band Gap Opening............................10

1.6.2 Chemical Substitution Doping..................................10

1.6.3 Graphene Nanoribbons.........................................11

1.7 Carbon Nanocages..................................................... 12

1.8 Functionalization of Graphene....................................... 13

$1.9 \mathrm{CF}_{\mathrm{x}}$ Batteries........................................................ 15

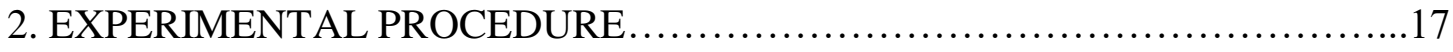

2.1 Hypothesis........................................................ 17

2.2 Synthesis of CNCs................................................... 17 
2.2.1 Precursor

2.2.2 Annealing.......................................................

2.2.3 Etching...................................................... 19

2.3 Functionalization of CNCs by Fluorination..............................19

2.4 Lithium/ $\mathrm{CF}_{\mathrm{x}}$ Primary Battery Fabrication..............................21

2.4.1 $\mathrm{CF}_{\mathrm{x}}$ Electrode Fabrication......................................... 21

2.4.2 Electrolyte Fabrication........................................... 21

2.4.3 Battery Assembly.............................................21

2.5 Discharge Capacity Testing.......................................24

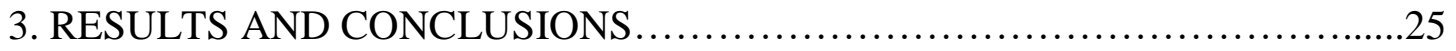

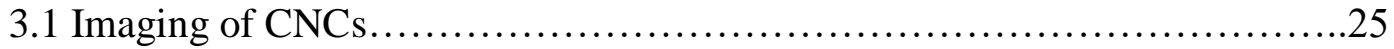

3.2 Raman and XPS Data for Fluorinated CNCs............................25

3.3 First Cycle Discharge Capacity Measurements...........................27

3.4 Conclusions.......................................................28

REFERENCES .......................................................29

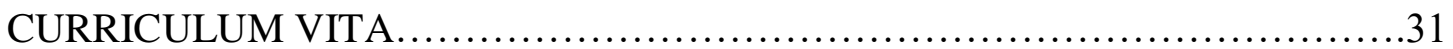




\section{LIST OF FIGURES}

FIGURE

PAGE

1.1 Configuration of Electrons in Carbon.......................................2

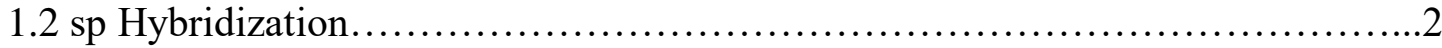

$1.3 \mathrm{sp}^{2}$ Hybridization.........................................................

$1.4 \mathrm{sp}^{3}$ Hybridization.....................................................4

1.5 Graphene as a Fundamental Structure.......................................5

1.6 Graphene Hexagonal Lattice and Reciprocal Lattice...........................6

1.7 Honeycomb Lattice Electronic Dispersion.....................................

1.8 N Doped Graphene with Electrical Properties..................................10

1.9 Fabrication of GNRs........................................................

1.10 Graphene Before and After Hydrogenation................................14

2.1 Annealing Schematic.................................................. 17

2.2 Programmed Furnace Temperature........................................ 18

2.3 Plasma System.........................................................20

2.4 Argon Glove Box....................................................22

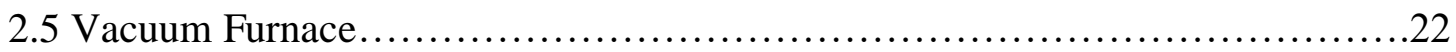

2.6 Coin Cell Batteries.................................................... 23

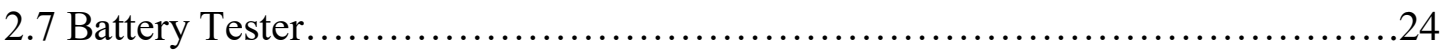

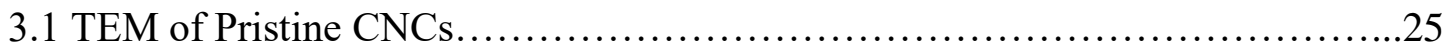

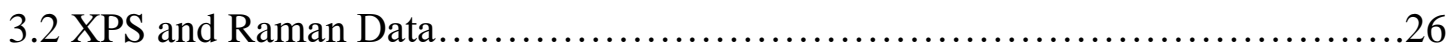

3.3 Voltage vs Discharge Capacity.......................................27 


\section{CHAPTER 1: INTRODUCTION AND BACKGROUND}

\subsection{Introduction}

Technology has developed rapidly in the past century. It has led to the creation of many new devices that rely on electricity to function. With the ever increasing demand for electricity, it becomes necessary to search for new, more efficient methods to meet this demand. One such area where the development of a more effective power source is that of primary batteries. An example of a case where such batteries are needed is for use in pacemakers. The more charge that can be stored in the same size battery the better. Cost is also always a consideration. As such, the goal for research in the field of improving primary batteries should be to develop a battery whose components and fabrication method are relatively inexpensive, while possessing high energy density, and strong stability, both in terms of storage life and safety.

\subsection{Electron Orbitals of Carbon}

Carbon, known as the primary building block of life, is an abundant element that is a prime candidate for use in devices. Carbon has 6 electrons arranged in the $1 s^{2} 2 s^{2} 2 p^{2}$ orbitals when in its ground state. The two electrons in the $1 \mathrm{~s}$ orbital are considered to be close enough to the nucleus of the atom that they are negligible in terms of consideration for chemical reactions. The other 4 electrons occupy the $2 \mathrm{~s}$ and $2 p$ orbitals, which are considered the outer orbitals, which are of significance in chemical reactions. In its ground state, two of the electrons remain in the 2 s orbitals while the other two occupy the two of the three $2 p$ orbitals (Figure 1.1 Upper). The 


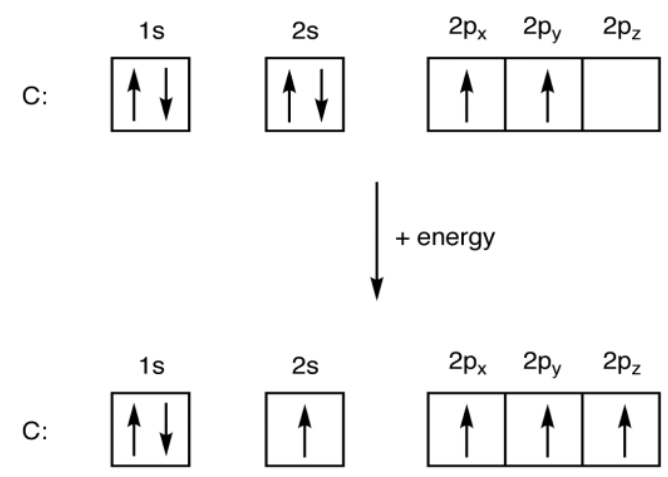

Figure 1.1: Configuration of electrons in carbon while in the ground state (Upper), and in the first excided state (Lower).

energy difference between the $2 \mathrm{~s}$ orbital and the $2 \mathrm{p}$ orbitals $\left(2 \mathrm{p}_{\mathrm{x}}, 2 \mathrm{p}_{\mathrm{y}}\right.$, and $\left.2 \mathrm{p}_{\mathrm{z}}\right)$ is approximately $4 \mathrm{eV}$. When a carbon atom comes in contact with other atoms, such as hydrogen, oxygen, or other carbon atoms, it becomes energetically favorable for the second electron in the 2 s orbital to jump up to the third $2 p$ orbital, due to the energy gain from the resulting covalent bond (Figure 1.1 Lower). This is a theoretical step, not one seen in experiments. This leads to a situation in which the carbon atom has four equivalent quantum mechanical states, $|2 \mathrm{~s}\rangle,\left|2 \mathrm{p}_{\mathrm{x}}\right\rangle,\left|2 \mathrm{p}_{\mathrm{y}}\right\rangle$, and $\left|2 \mathrm{p}_{\mathrm{z}}\right\rangle$. The superposition of the $\mid 2 s>$ state with $n\left|2 p_{j}\right\rangle$ is referred to as $s p^{n}$ hybridization, an important part of covalent carbon bonds [7].

\subsection{1 sp Hybridization}

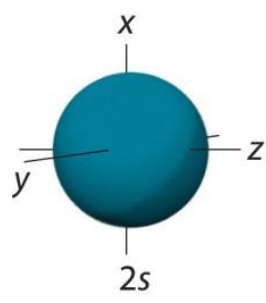

$2 s$
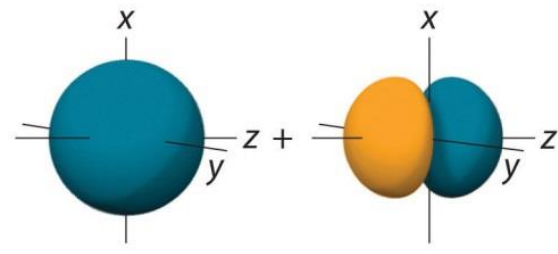

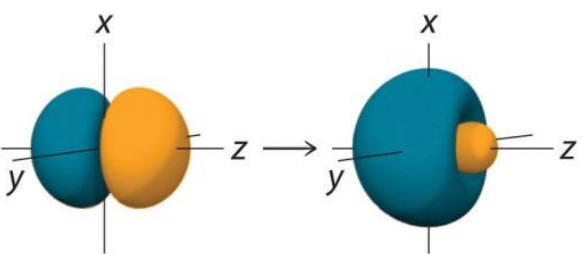

sp hybrids

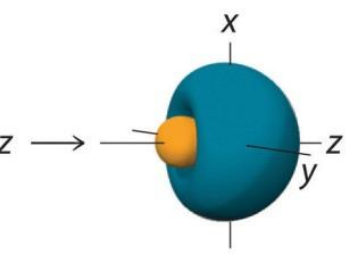

Figure 1.2: Illustration of sp hybridization [1]. 
sp hybridization is the result of the $|2 s\rangle$ state mixing with one of the three $2 p$ orbitals, for the sake of illustration, consider the $\left|2 p_{x}\right\rangle$ state. By taking the symmetric and anti-symmetric combinations of the original two states, the resultant states are,

$$
\begin{aligned}
& \left|s p_{+}\right\rangle=\frac{1}{2}\left(|2 s\rangle+\left|2 p_{x}\right\rangle\right) \\
& \left|s p_{-}\right\rangle=\frac{1}{2}\left(|2 s\rangle-\left|2 p_{x}\right\rangle\right)
\end{aligned}
$$

Meanwhile the $\left|2 p_{y}\right\rangle$ and $\left|2 p_{z}\right\rangle$ states remain the same, unaffected by the superposition. An example of this hybridization is the acetylene molecule $\mathrm{H}-\mathrm{C} \equiv \mathrm{C}-\mathrm{H}$, in which the sp orbitals of the carbon atoms form a $\sigma$ bond, with each other while also bonding with their respective hydrogens. This $\sigma$ bond is significantly stronger than the $\pi$ bonds formed by the other $2 p$ orbitals that make up the remaining two parts of the triple bond connecting the carbons.

\subsection{2 $\mathrm{sp}^{2}$ Hybridization}

$\mathrm{sp}^{2}$ hybridization is the result of the $2 \mathrm{~s}$ orbital being superposed on not one, but two of the $2 \mathrm{p}$ orbitals. Considering specifically the $\mathrm{sp}^{2}$ hybridization of the $|2 \mathrm{~s}\rangle$, $\left|2 p_{\mathrm{x}}\right\rangle$, and $\left|2 p_{\mathrm{y}}\right\rangle$ states, the resulting mechanical states are,

$$
\begin{aligned}
& \left|\mathrm{sp}_{1}^{2}\right\rangle=\frac{1}{\sqrt{3}}|2 \mathrm{~s}\rangle+\sqrt{\frac{2}{3}}\left|2 \mathrm{p}_{\mathrm{y}}\right\rangle \\
& \left|\mathrm{sp}_{2}^{2}\right\rangle=\frac{1}{\sqrt{3}}|2 \mathrm{~s}\rangle+\sqrt{\frac{2}{3}}\left(\frac{\sqrt{3}}{2}\left|2 \mathrm{p}_{\mathrm{x}}\right\rangle+\frac{1}{2}\left|2 \mathrm{p}_{\mathrm{y}}\right\rangle\right) \\
& \left|\mathrm{sp}_{3}^{2}\right\rangle=-\frac{1}{\sqrt{3}}|2 \mathrm{~s}\rangle+\sqrt{\frac{2}{3}}\left(-\frac{\sqrt{3}}{2}\left|2 \mathrm{p}_{\mathrm{x}}\right\rangle+\frac{1}{2}\left|2 \mathrm{p}_{\mathrm{y}}\right\rangle\right)
\end{aligned}
$$


This results in the $\mathrm{sp}^{2}$ orbitals all being oriented along the same plane, while the remaining $\mathrm{sp}_{z}$ orbital is orthogonal to said plane. The angle between these orbitals is $120^{\circ}$, as seen in Figure 1.3 below.

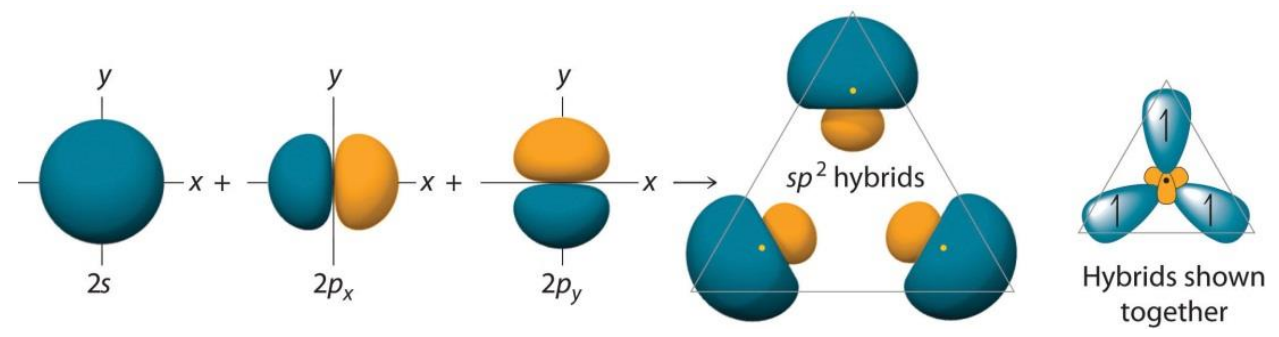

Figure 1.3: Illustration of $\mathrm{sp}^{2}$ hybridization [1].

An example of $\mathrm{sp}^{2}$ hybridization is a benzene ring. In a benzene ring, 6 carbon atoms are linked together in a ring by $6 \sigma$ bonds, forming a hexagonal shape, while also bonding with their respective hydrogen atoms. The $2 \mathrm{p}$ orbitals that are not a part of the $\mathrm{sp}^{2}$ hybridization form three $\pi$ bonds. These bonds are the second bonds creating the alternating double bonds in the hexagon. The single and double carboncarbon bonds in benzene are roughly the same length.

\subsection{3 $\mathrm{sp}^{3}$ Hybridization}

The final hybridization of carbon, $\mathrm{sp}^{3}$, is achieved by superposing the $2 \mathrm{~s}$ and the three $2 p$ orbitals. In this case, the resulting orbitals form a 3 dimensional tetrahedron, where each bond is offset from every other bond by an angle of $109.5^{\circ}$, as seen in Figure 1.4.

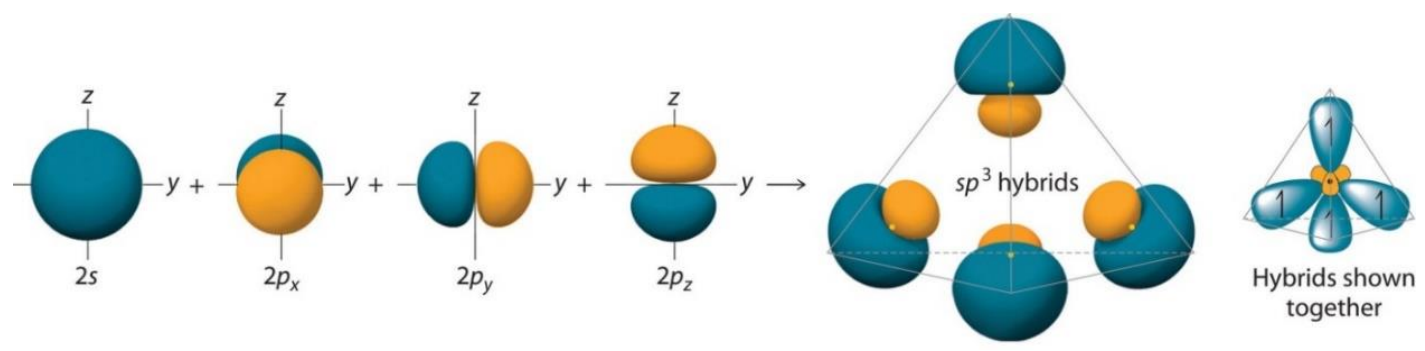

Figure 1.4: Illustration of $\mathrm{sp}^{3}$ hybridization [1].

An example of $\mathrm{sp}^{3}$ hybridization is Methane, in which each of the hybridized orbitals form a bond with a hydrogen atom. $\mathrm{sp}^{3}$ hybridized bonds are also found in 
diamonds. The structure of diamonds is known as a diamond lattice, and it consists of two face center cubic lattices shifted off of one another by $1 / 4$ the diagonal length of the lattice. While both are made up solely of carbon, the fact that diamond is bonded together by strong $\sigma$ bonds that are not easily broken make it both a strong material, as well as a good insulator. Graphite on the other hand has a layered structure, making it soft. It is held together by $\pi$ bonds, which have delocalized electrons, making it an effective conductor.

\subsection{Discovery of Graphene}

While having been hypothesized since 1947 [8], graphene was considered to be nothing more than a theoretical material until quite recently. In fact it was not until 60 years later that free standing graphene was discovered [2]. Graphene is a single atom thick, and is thus considered to be a 2 dimensional material. In said 2

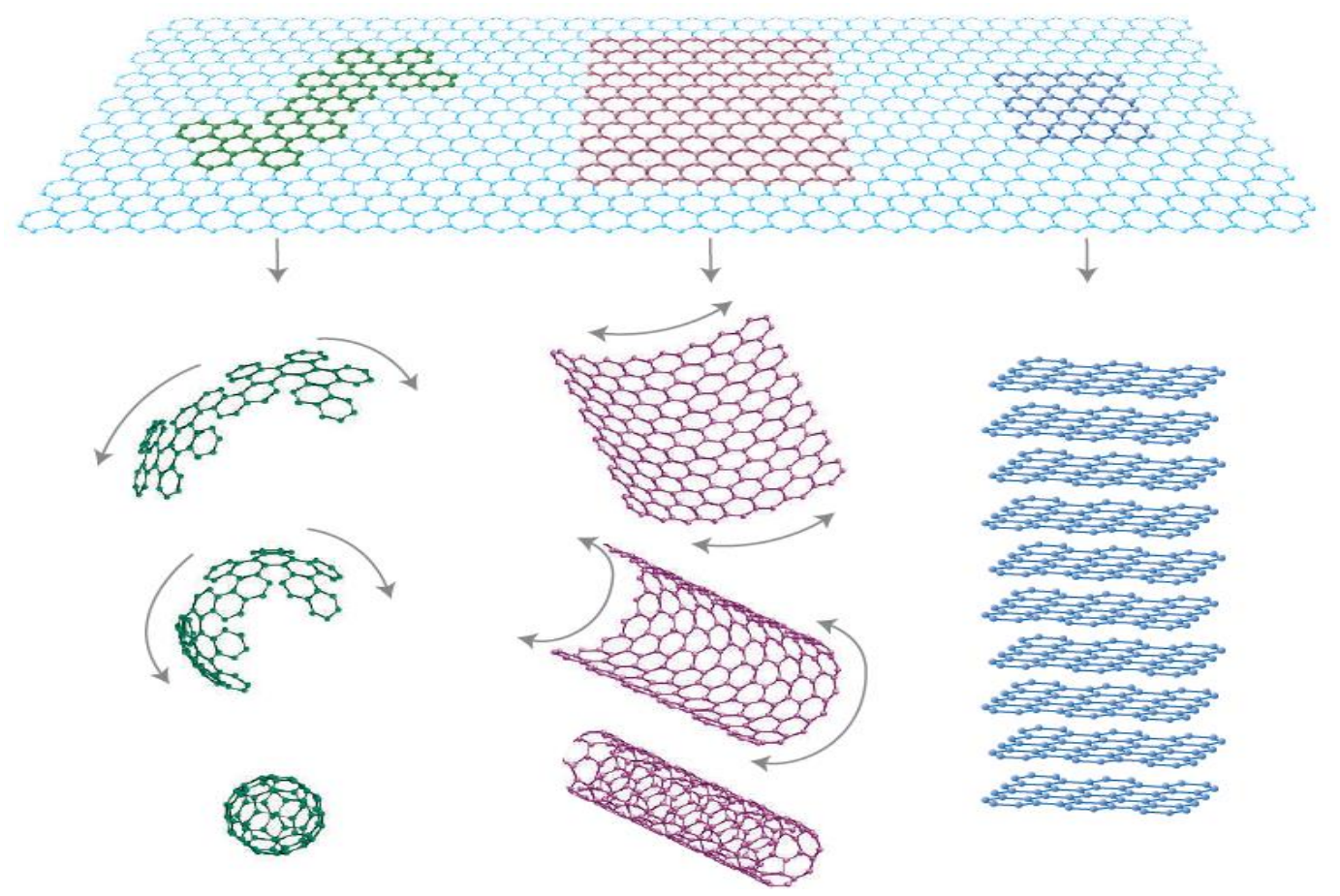

Figure 1.5: Examples of how graphene can be used to create more complex materials including Buckminsterfullerene (left), Carbon Nanotubes (center), and Graphite (right) [2]. 
dimensions it has a honeycomb lattice, and is considered to be the most fundamental graphitic structure, which can be manipulated to create other graphitic materials. It can be wrapped into spheres called buckminsterfullerene, rolled into nanotubes, or stacked into graphite, as can be seen in Figure 1.5. When there are 1-2 layers of graphene present, the material is considered to have zero band gap, however, as more layers are added, complications arise in the spectra. Once the number of layers exceeds 10, the material acts largely the same as a thin film of graphite [9], and as such are no longer considered to be 2 dimensional.

\subsection{Band Structure of Graphene}

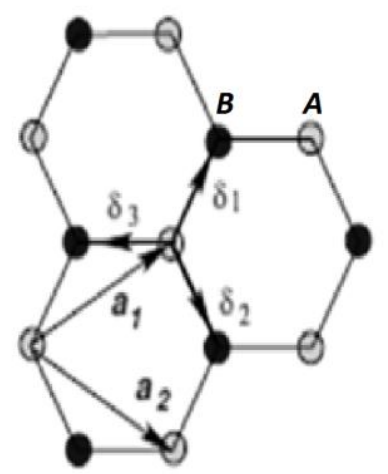

(a)

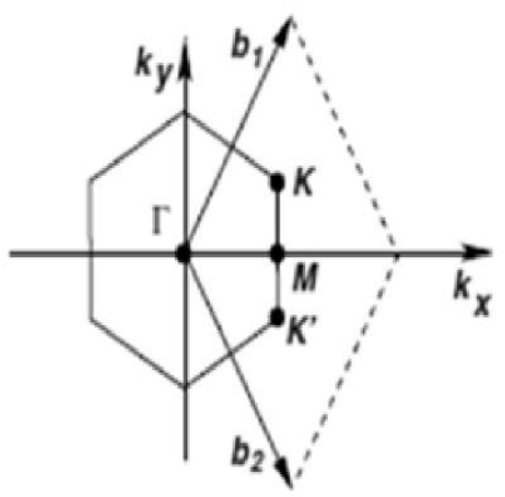

(b)

Figure 1.6: (a) Graphene hexagonal lattice, (b) Reciprocal lattice of graphene, with the first Brillouin zone denoted by the hexagon. [3]

The tight binding method, first introduced by Wallace [8], has become the predominant method for graphene band structure analysis. Graphene is of great interest as a material due to the fact the first two bands neither overlap, nor have a gap separating them, but rather intersect at what are known as Dirac points. At these Dirac points is where the Fermi level is found, making graphene a semiconductor with zero band gap. A hexagonal lattice of graphene can be seen in Figure 1.6a, along with the reciprocal lattice and first Brillouin zone in Figure 1.6b. On the (a) side of the figure, 
the lattice is formed by superposing two triangular lattices, labeled as A and B. The basis vectors for triangle lattice A are $\boldsymbol{a}_{i}$ and the B triangle lattice is shifted by nearest neighbor vectors $\delta_{i}$ where $\mathrm{i}=1,2,3$. Notable in the (b) side of the figure is that the above mentioned Dirac points are found at the points K and K'. In these diagrams, the carbon to carbon distance is,

$$
a=0.142 \mathrm{~nm}
$$

$\boldsymbol{a}_{1}$ and $\boldsymbol{a}_{2}$ are then,

$$
\begin{aligned}
& \overrightarrow{a_{1}}=\frac{a}{2}(3, \sqrt{3}) \\
& \overrightarrow{a_{2}}=\frac{a}{2}(3,-\sqrt{3})
\end{aligned}
$$

Then $\boldsymbol{\delta}_{1}, \boldsymbol{\delta}_{2}$, and $\boldsymbol{\delta}_{3}$ are,

$$
\begin{aligned}
& \vec{\delta}_{1}=\frac{a}{2}(1, \sqrt{3}) \\
& \vec{\delta}_{2}=\frac{a}{2}(1,-\sqrt{3}) \\
& \vec{\delta}_{3}=-a(1,0)
\end{aligned}
$$

$\boldsymbol{b}_{1}$ and $\boldsymbol{b}_{2}$ can then be found to be,

$$
\begin{aligned}
& \vec{b}_{1}=2 \pi \frac{\vec{a}_{2} \times \hat{z}}{A}=\frac{2 \pi}{3 a}(1, \sqrt{3}) \\
& \vec{b}_{2}=2 \pi \frac{\vec{a}_{1} \times \hat{z}}{A}=\frac{2 \pi}{3 a}(1,-\sqrt{3})
\end{aligned}
$$

Where $\mathrm{A}$ is the unit cell area. Clearly, since each triangular lattice contains a single carbon atom, a unit cell of graphene contains two carbon atoms. Of carbon's 4 valence electrons, 3 are bonded to the adjacent carbon atoms with strong $\sigma$ bonds. These bonds do not contribute to electronic conduction. The $4^{\text {th }}$ electron of each carbon atom however is the $2 \mathrm{p}_{z}$ orbital, which is oriented orthogonally to the plane of the hexagonal lattice. These electrons should each be forming a $\pi$-bond. One of these 
bonds, is called $\pi$, and refers to a valence band bond, while the other, called $\pi^{*}$ refers to a conduction band anti-bond. Given that electrons can travel through graphene to either their nearest, or next-nearest neighbor, the tight binding Hamiltonian to the first order is given as, [3]

$$
H=-t \sum_{<i, j>, \sigma} a_{\sigma, \mathrm{i}}^{\dagger} b_{\sigma, \mathrm{j}}-t^{\prime} \sum_{\ll i, j\rangle, \sigma}\left(a_{\sigma, \mathrm{i}}^{\dagger} a_{\sigma, \mathrm{j}}+b_{\sigma, \mathrm{i}}^{\dagger} b_{\sigma, \mathrm{j}}\right)
$$

Where $\mathrm{t}$ is approximately $2.8 \mathrm{eV}$ and $\sigma$ refers to the spin of the electron. Thus, the energy bands are derived to be,

$$
E_{ \pm}= \pm t \sqrt{3+f(\boldsymbol{k})}-t^{\prime} f(\boldsymbol{k})
$$

Where $\mathrm{f}(\mathbf{k})$ is,

$$
f(\boldsymbol{k})=2 \cos \left(\sqrt{3} k_{y} a\right)+4 \cos \left(\frac{\sqrt{3}}{2} k_{y} a\right) \cos \left(\frac{3}{2} k_{x} a\right)
$$

And thus, the energy dispersion is,

$$
E_{ \pm}(\boldsymbol{k})= \pm v_{F}|\boldsymbol{k}|
$$

Where $v_{F}$ is the Fermi velocity, and is given by,

$$
v_{F}=\frac{3 t a}{2}
$$

The positive energy term refers to the $\pi^{*}$ bond and the negative term refers to the $\pi$ bond. This energy dispersion can be seen in Figure 1.7.

\subsection{Synthesis of Graphene}

There are multiple techniques used to generate graphene, each with their benefits and drawbacks. The first method developed is that of mechanical exfoliation [2]. This method involves peeling graphene flakes off of highly oriented pyrolytic graphite. While the resulting flakes of graphene generated by this method are of a high quality, the method does not produce enough graphene, both in terms of size and quantity, to be useful in industry. Additionally, this method cannot be scaled in such a way to correct this problem. Another method worth noting is that of forming graphene 


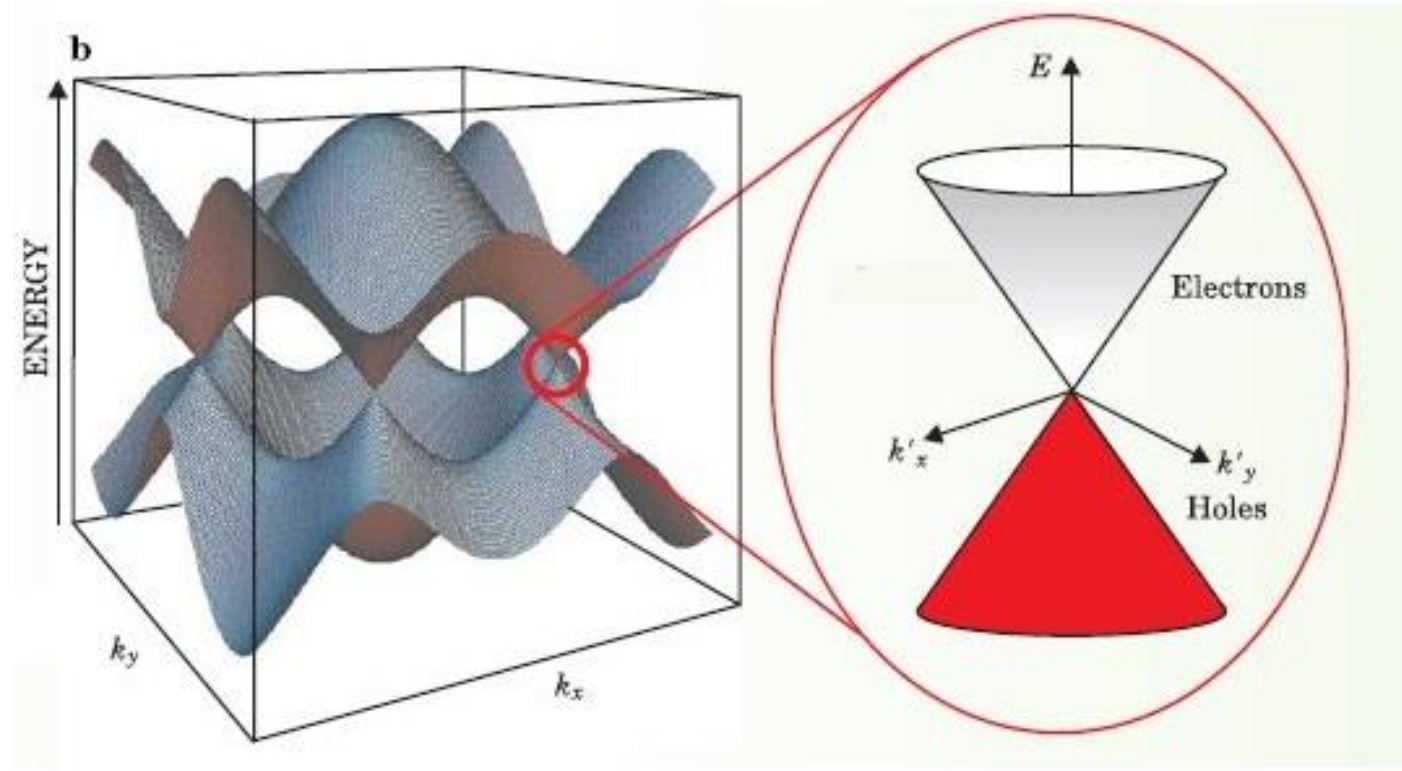

Figure 1.7: Honeycomb lattice electronic dispersion for $\mathrm{t}=2.7 \mathrm{eV}$. [3]

epitaxially on silicon carbide [10]. This method is appealing due to silicon carbide being commercially sold, and due to the fact that the resulting graphene is formed on a surface where it can be tested immediately, thanks to the electrical properties of silicon. A downside of this method is that it requires strong vacuum. Unfortunately, the quality of the resulting graphene was not usable in devices. In order to obtain higher quality graphene, temperatures can be increased [11]. This however leads to the major limitations of this method. First, the ingredients are costly, and second, the high temperatures needed are restrict the potential applications. Chemical Vapor Deposition (CVD) is another method, similar to epitaxial growth, except that graphene is grown on thin metallic films. It is however, notably different in that it does not require as strict conditions, such as strong vacuum, alleviating some of the problems with epitaxial growth. The potential to texture the metallic film to improve the quality of the resulting graphene is also appealing [12].

\subsection{Engineering a Band Gap in Graphene}

While graphene is a promising candidate to eventually replace silicon in many ways, the fact that it has zero band gap is a major drawback in applications such as 
transistors. As such, methods to alleviate this issue by manipulating graphene into having a band gap are a major area of research [12].

\subsubsection{Substrate Induced Band Gap Opening}

Early attempts to generate a band gap in graphene included utilizing the effect of the substrate. By adding a coating of h-BN between the graphene and nickel, a 0.5 $\mathrm{eV}$ band gap was measured [13]. There are also theoretical studies that support the claim that this method can produce a band gap $[14,15]$. Given this, it is possible to use a substrate to impose a band gap, however these band gaps are not necessarily great enough to produce the desired results, in terms of usability in electronic devices.

\subsubsection{Chemical Substitution Doping}
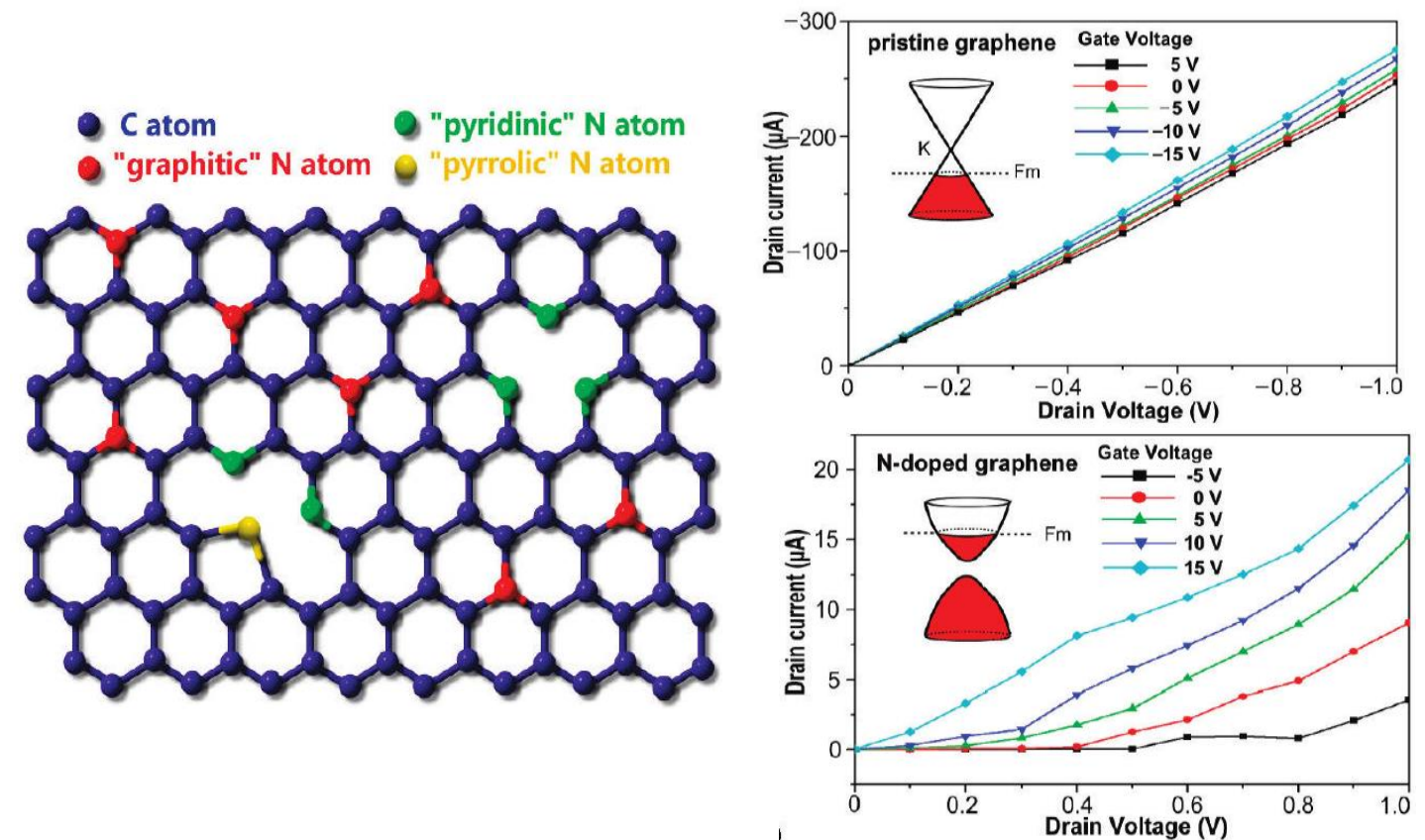

Figure 1.8: $\mathrm{n}$-doped graphene with $\mathrm{N}$ atoms(left), pre- and post-doping electrical properties of sample. [5]

Chemical substitution doping is a prominent method of doping conventional semiconductors. This same method can be used to dope graphene. Substituting nitrogen atoms for some of the carbon atoms in a graphene lattice, for example, can lead to $\mathrm{n}$ type doping [5]. Similarly, by substituting boron, $\mathrm{p}$ type doping can be 
achieved [16]. Theoretically impurities in the graphite favor positions in the lattice far from other impurities. Additionally, the doping atoms energetically prefer to occupy edge sites in the lattice. This means that the resulting samples tend towards more even distribution of impurities in the center of the sample, provided the sample is doped evenly, with added concentration of impurities along the edges. $\mathrm{n}$ and $\mathrm{p}$ type doping has been achieved by such methods as CVD as well as the arc discharge method. It has been observed that the resulting graphene has the desired band gap, as can be seen in Figure 1.8. There is one major drawback to the use of dopants in graphene. One of the major attractions of pristine graphene is that it possesses excellent electric properties, such as carrier mobility and conductivity. With the introduction of impurities to the graphene, these electric properties are inferior to those of pristine graphene. Even taking this into account however, the nonzero band gap of the resulting sample makes the process of chemical substitution doping worthwhile.

\subsubsection{Graphene Nanoribbons}

While above mentioned methods have been successful to varying degrees in producing nonzero band gap graphene, they do all demand that the 2 dimensional nature of graphene be preserved and that the band gap be generated without compromising this feature. Another approach is that of the Graphene Nanoribbon (GNR). Instead of relying on the 2 dimensional properties of graphene as is, this method starts by rolling a sheet of graphene into a nanoribbon, or creating said ribbon starting from a Multiwalled carbon nanotube (MWCNT) and peeling back layers until all that is left is a GNR, as seen in Figure 1.9. By doing this, the electrons of the graphene are confined to the effectively 1 dimensional line along which the GNR runs. Notable about this method is that no impurities are added to the graphene lattice in order to generate GNRs and as such, the highly desirable electric properties of 


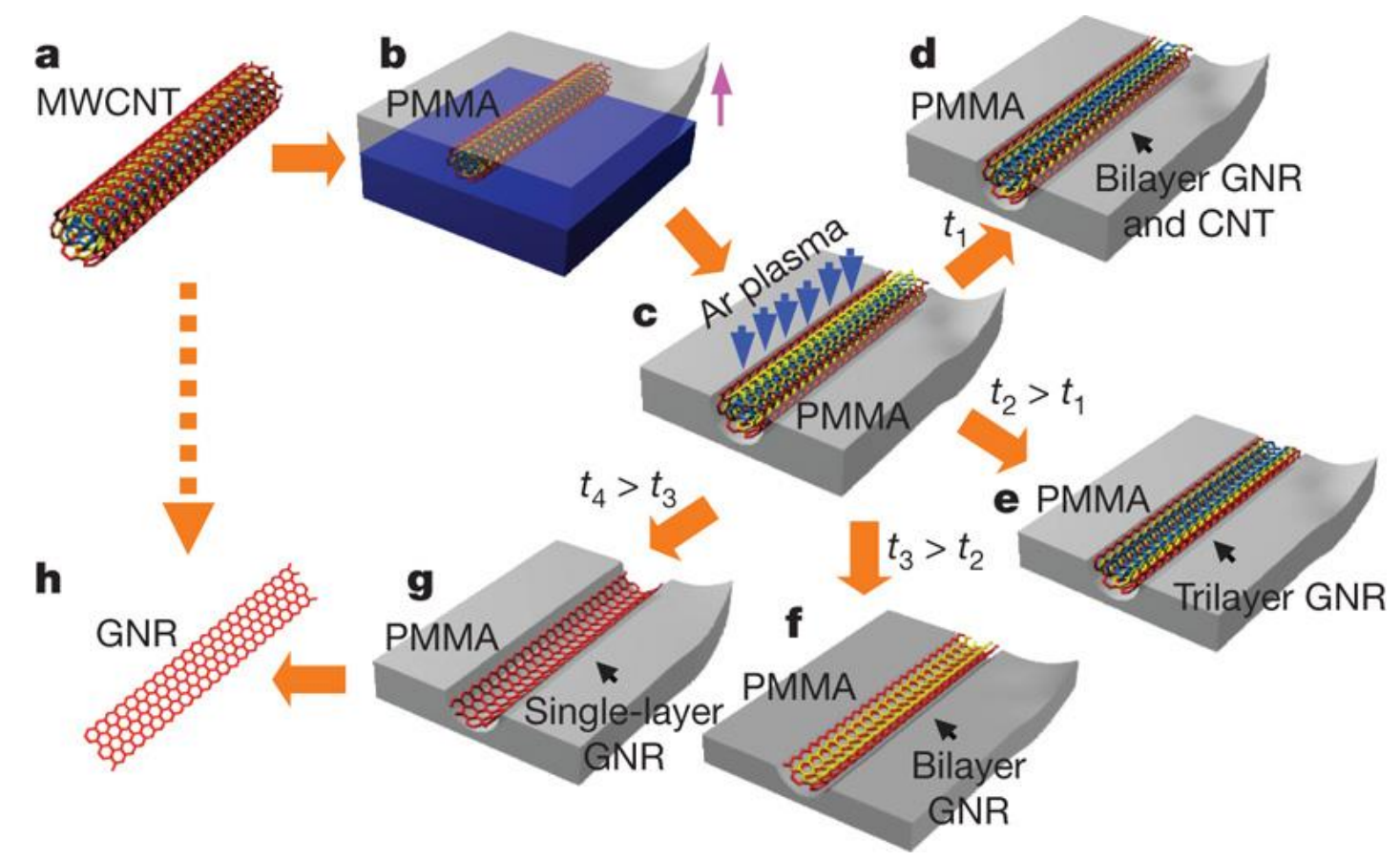

Figure 1.9: Fabrication of GNRs (a) pristine MWCNT precursor. (b) MWCNT deposited on silicon, and then coated with PMMA film. (c) PMMA-MWCNT film removed from silicon substrate, flipped over, and exposed to argon plasma. (d through g) Products depend on time of etching, where (d) was etched for the shortest time, and (g) the longest. (h) After removal of the PMMA the result is the desired GNR. [6]

graphene remain largely intact. It has been observed that these GNRs have both a finite band gap as well as strong carrier mobility. They are also demonstrated to exhibit electronic confinement, coherence, and Dirac nature of carriers [17].

\subsection{Carbon Nanocages}

In recent years, Carbon Nanocages (CNCs) have become a topic of great interest due to their potential usefulness in many applications, nano-electronicmagnetic devices, superconductors, gas storage, biotechnology, nano-transportation, and more [18]. Also of interest is that should small enough CNCs be fabricated, they could essentially be considered to be multilayered Buckminsterfullerene, which is considered to be a 0 dimensional material in the same way that GNRs are considered 
to be 1 dimensional [2]. In addition to this, the fact that CNCs are curved into the shape of a sphere necessarily means that they have no edges. This is significant in that graphene and GNRs both have edges to their structure, which lead to the need for either approximations or more complex models to take these edges into account. CNCs have no such drawback. There are various methods available to synthesize $\mathrm{CNCs}$, and the resulting particles can be tailored to suit desired properties depending on what method is used. The general method for generating CNCs consists of heating a precursor containing both a catalyst material, such as nickel or iron, and a carbon material to provide the graphitic layers. The precursor is then converted to CNCs formed around catalyst cores, usually by heating. The catalyst is then removed, and what remains in the hollow CNC, ready for use. In 2014, Wang et al. observed the formation of CNCs grown on iron. They report that when preparing their sample at $700^{\circ} \mathrm{C}$ they observed CNCs of 10-50 nm outer diameter, and of shell thickness 2-5 nm. At $1000^{\circ} \mathrm{C}$ the size of the nanocages had increased to $20-50 \mathrm{~nm}$ with a thickness of 3-10 nm. Finally at $1500^{\circ} \mathrm{C}$ they report nanocages of $50-100 \mathrm{~nm}$ size with a thickness of 5-15 nm [19]. Clearly then, their technique allows for the selection of the size and thickness of resulting CNCs. Other groups have used magnesium oxide as their template particle, in the process achieving a specific surface area of $997 \mathrm{~m}^{2} \mathrm{~g}^{-1}$ and micro-meso pore volume of $3.307 \mathrm{~cm}^{3} \mathrm{~g}^{-1}$ [20]. The major issue limiting the usefulness of $\mathrm{CNCs}$ is that the processes needed to yield high quality results can be expensive, and often require conditions not fit for industry. Recently however, a novel method for synthesizing CNCs has been reported allowing for the bypassing these limitations [21]. Due to these recent advances this field of research shows a great deal of promise.

\subsection{Functionalization of Graphene}




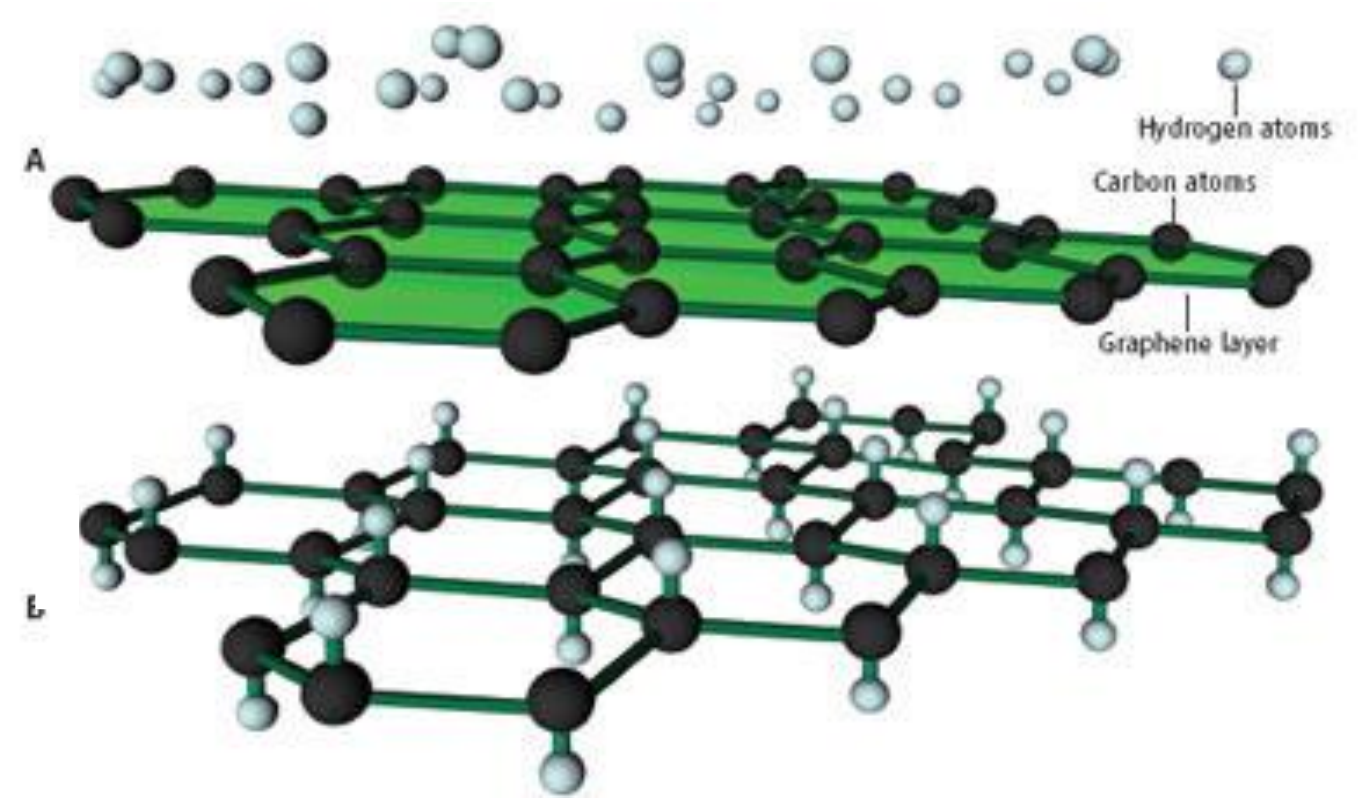

Figure 1.10: Graphene before hydrogenation (top). Graphene after hydrogenation (bottom). [4]

Properties such as electron confinement effects, high carrier mobility, and a tunable band gap have propelled graphene to significance in many fields of research [2]. Some of the fields in which graphene can be applied include field-effect transistors, lithium ion batteries, and super capacitors [22]. In order to make graphene useful in these applications, it is often necessary to modify it. The process of altering the surface chemistry of graphene to allow it to be used for new purposes, called functionalization, is one of the primary methods utilized in altering its physical and mechanical properties [23]. There are two types of functionalization for graphene. Covalent functionalization involves the removal of the carbon atoms from the graphene lattice, and their replacement with a different element. This can be seen in Figure 1.8, where nitrogen acts as the replacement. This requires damaging the structure of the graphene, which in turn weakens the electric properties, as discussed previously. Noncovalent functionalization, on the other hand, does not involve any alteration to the graphene lattice $\sigma$ bonds. Instead, the free $\pi$ bonds that exist perpendicular to the plane of graphene bond with the newly introduced 
functionalizing element, effectively changing the hybridization of the lattice from $\mathrm{sp}^{2}$ to $\mathrm{sp}^{3}$ as can be seen in Figure 1.10. It is expected that this rehybridization will induce the formation of a band gap [24]. Three common choices for graphene functionalization are hydrogen — as seen in Figure 1.10 — oxygen, and fluorine. In oxidized graphene the oxygen atoms damage the graphene structure enough to lower the conductivity and carrier mobility by orders of magnitude [25]. On the other hand, in hydrogenated graphene while the lattice is not damaged, it begins to lose hydrogen atoms when heated, this makes hydrogenated graphene undesirable in many applications where high temperatures are necessary. Fluorine, like hydrogen, bonds with graphene noncovalently, and as such does not damage the graphene lattice structure. In addition to this, unlike hydrogen, fluorine binds strongly with carbon, and as such is thermodynamically stable. In addition, fluorine is the most electronegative element, leading to great interest in fluorinated graphene for use in devices such as batteries.

\section{9 $\mathrm{CF}_{\mathrm{x}}$ Batteries}

Carbon fluoride $\left(\mathrm{CF}_{\mathrm{x}}\right)$ is a promising material for use as a cathode in lithium ion batteries. It boasts a good safety record, high energy density, a long storage life, low self-discharge, and a wide temperature operating range [26]. These batteries have garnered interest in fields ranging from medicine [27] to the military [28]. The fact that the cathode is tunable allows for the optimization of the properties of the battery to ensure it is ideal for use in whatever the desired situation may be. The drawbacks of $\mathrm{CF}_{\mathrm{x}}$ cathodes are their low power drain and poor conductivity, limiting their usefulness in high-power applications [29]. While fabrication methods have been more expensive than other cathodes, $\mathrm{CF}_{\mathrm{x}}$ electrodes are free from many of the major 
drawbacks of other cathodes, giving them a great deal of potential for continued experimentation to allow for more varied applications. 


\section{CHAPTER 2: EXPERIMENTAL PROCEDURE}

\subsection{Hypothesis}

It has been discussed previously that there is great demand for low cost stable primary batteries with high energy density. In order to achieve this, the potential use of a nickel acetate / citric acid precursor, annealed to form CNCs, and then fluorinated to form $\mathrm{CF}_{\mathrm{x}}$ for use in lithium ion primary batteries is proposed and tested. It is expected that the CNCs will allow for strong electric properties, while the fluorine will serve to open the band gap while ensuring the product remains stable.

\subsection{Synthesis of CNCs}

\subsubsection{Precursor}

The precursor used for this experiment was a 1 to 1 weight ratio powder mixture of nickel acetate and citric acid. When heated the nickel of the nickel acetate breaks free and becomes the template, or catalyst, while the citric acid provides the carbon necessary to grow the graphene around said template.

\subsubsection{Annealing}

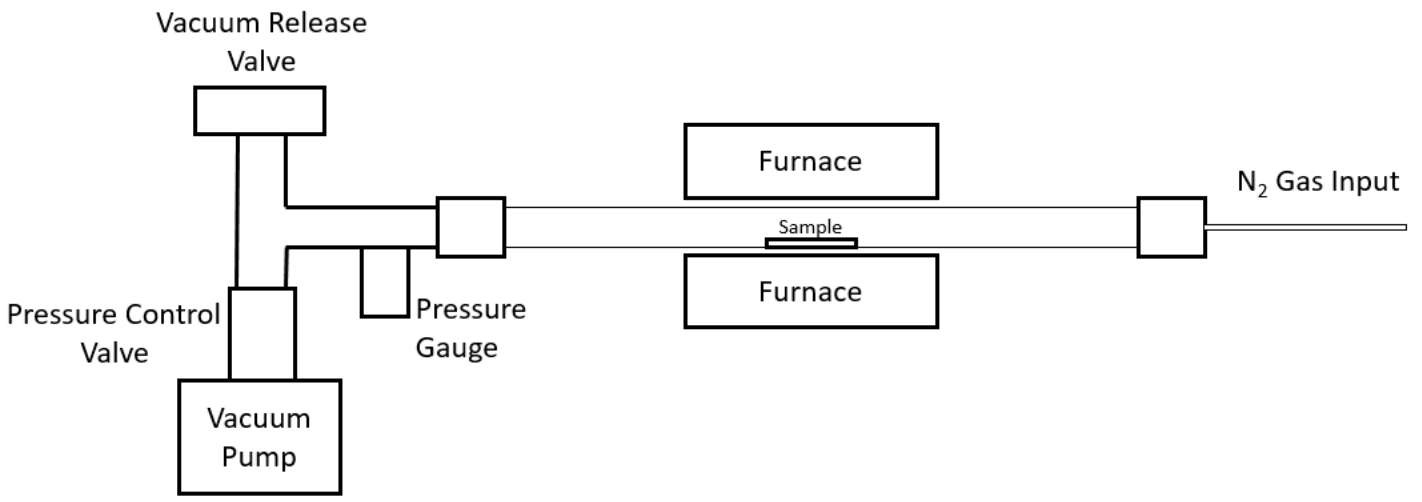

Figure 2.1: Schematic of experimental setup for annealing of the precursor. 
Annealing of the precursor was accomplished by way of a Thermolyne ${ }^{\mathrm{TM}}$ 21100 Tube Furnace, as illustrated in Figure 2.1. First, the sample was loaded evenly into a $10 \mathrm{~cm}$ long ceramic crucible. The crucible was then inserted into a quartz tube and pushed into the tube furnace until it was measured to be located exactly at the center of the tube furnace. The end of the quartz tube was then sealed and a vacuum pump was turned on. The system was then pumped down to 200 mTorr in order to remove any atmospheric contaminants, such as oxygen, from the system. The speed at which the system was pumped down was controlled by the pressure control valve in order to minimize loss of sample. After this, $\mathrm{N}_{2}$ gas was introduced to provide an inert atmosphere to ensure that only the desired reaction took place. The tube furnace was then used to heat the sample at a rate of $50^{\circ} \mathrm{C} / \mathrm{min}$ up to $600^{\circ} \mathrm{C}$. The temperature was then held at $600^{\circ} \mathrm{C}$ for 10 minutes. After this the furnace was turned off allowing the sample to cool back down to room temperature. After the sample was at room temperature, the vacuum pump was turned off, and the seal was broken. The $\mathrm{N}_{2}$ gas was also turned off. The now-annealed sample was then retrieved and observed under

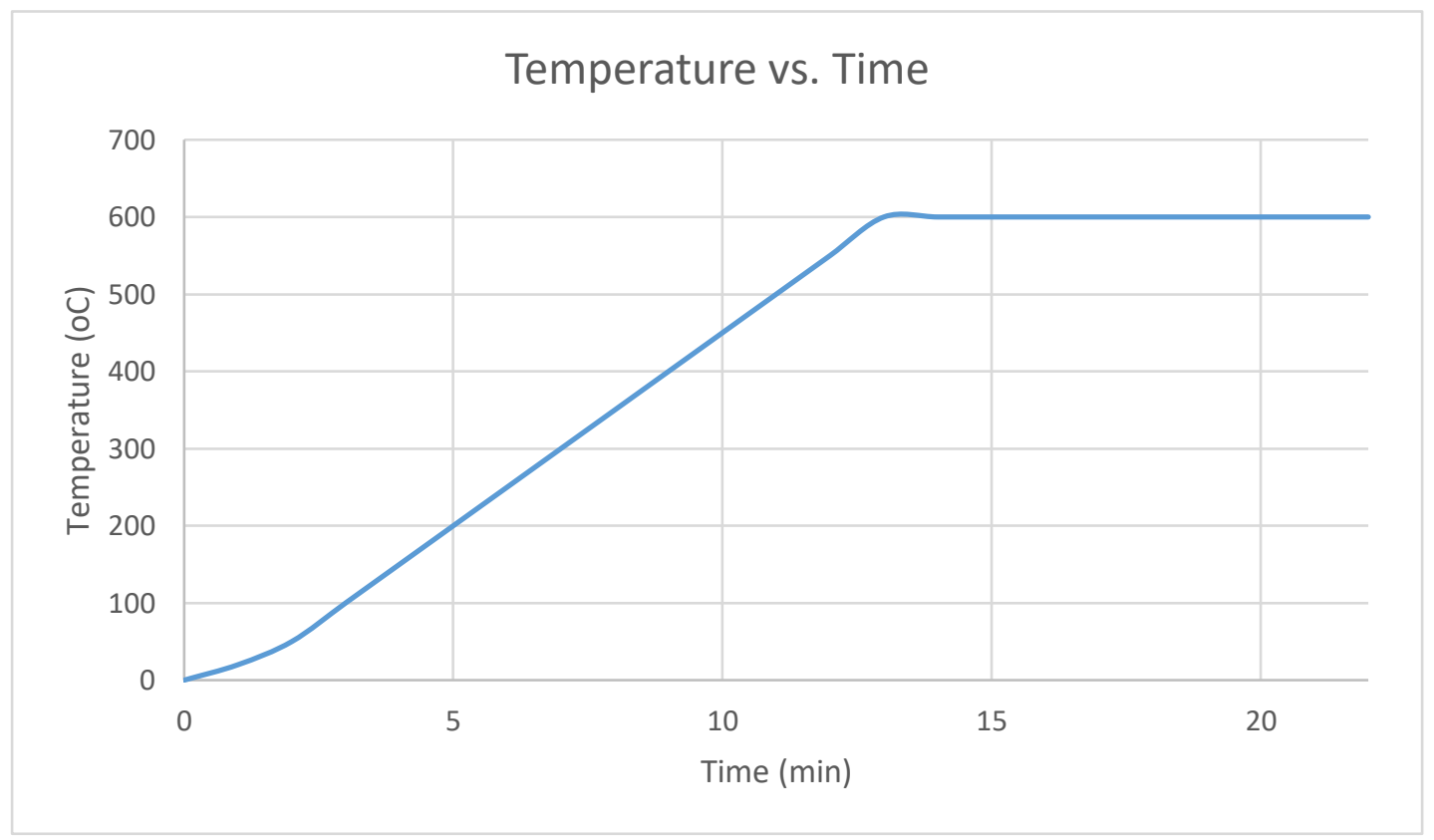

Figure 2.2: Programmed temperature in the tube furnace. 
a Transition Electron Microscope (TEM) to ensure the resulting particles were homogeneous and of a desirable size.

\subsubsection{Etching}

The sample was placed in concentrated nitric acid in order to remove the nickel particles from the centers of the resultant particles, leaving behind only the desired CNCs. The solution was stirred with a magnetic stirrer, and left overnight. The solution was then filtered to separate the CNCs from the nickel dissolved in nitric acid, and rinsed several times with deionized water. Finally, the filtered material was dried, now containing solely pristine CNCs.

\subsection{Functionalization of CNCs by Fluorination}

To functionalize the pristine $\mathrm{CNCs}$, a split-ring capacitively coupled radio frequency (RF) plasma system was used, as can be seen in Figure 2.3. The CNCs were loaded into a $10 \mathrm{~cm}$ long ceramic crucible as during annealing. The crucible was then placed at the center of the plasma system rings. The system was first slowly pumped down to a pressure of 250 mTorr with the vacuum pump regulated by the pressure control valve. Water flow was then supplied to the plasma system to function as a coolant. Tetrafluoromethane $\left(\mathrm{CF}_{4}\right)$ gas was then introduced to the system and the plasma system was turned on. The flow rate of $\mathrm{CF}_{4}$ gas was adjusted between $4 \mathrm{sccm}$ and $12 \mathrm{sccm}$ to maintain the strongest plasma possible for the experiment. Too much $\mathrm{CF}_{4}$ gas in the chamber would result in the plasma system failing to ionize fluorine particles, on the other hand, too little gas means that there is not enough fluorine present to bond with the CNCs at an optimal rate, even if all of the gas is ionized. The power supplied to the plasma system was varied from $300 \mathrm{~W}$ to $400 \mathrm{~W}$, in order to maintain the strong plasma for as long as possible without overheating the system. For longer duration fluorination samples, when the plasma began to weaken, after 2 to 4 
hours of fluorination, the plasma system and $\mathrm{CF}_{4}$ were turned off for 15 minutes to allow the plasma system to cool. While the sample was undergoing the fluorination, a vibrating motor was used to shake the sample, causing it to redistribute, ensuring that the entire sample was fluorinated uniformly. After the desired duration of fluorination was achieved, the motor, $\mathrm{CF}_{4}$ gas, and plasma system were turned off. The water was then shut off. Finally, the vacuum pump was turned off and the seal was broken in a controlled manner by way of the vacuum release valve. The fluorinated CNCs were then removed from the system, now ready for use in the fabrication of a $\mathrm{CF}_{\mathrm{x}}$ cathode. (a)
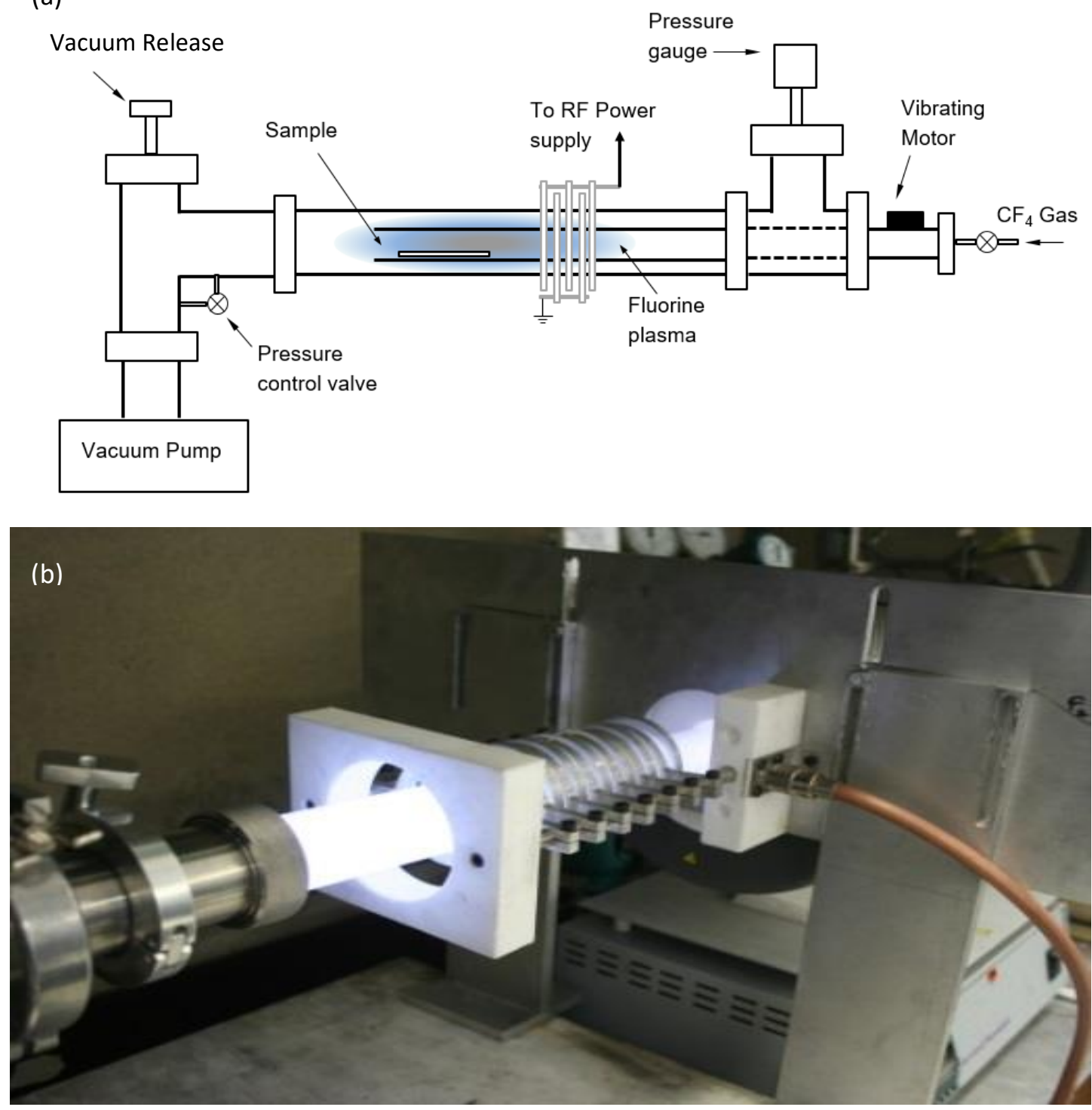

Figure 2.3: (a) Schematic of RF plasma system setup. (b) Image of plasma system in use. 


\subsection{Lithium/CF $\mathrm{x}_{\mathrm{x}}$ Primary Battery Fabrication}

\subsubsection{CF $\mathrm{x}$ Electrode Fabrication}

The $\mathrm{CF}_{\mathrm{x}}$ electrode was made of a combination of Fluorinated CNCs, and a carbon binder. Two different amounts of $\mathrm{CF}_{\mathrm{x}}$ were tested in batteries. The first being $5.0 \mathrm{mg}$ and the second being $3.0 \mathrm{mg}$. The amount of binder used in both cases was 3.0 mg. The ratio of fluorinated CNCs to binder was not considered important as the sole purpose of the binder was to conduct, and did not have a notable impact on the charge capacity of the resultant batteries. The CNCs and binder were both placed in a mortar. Two drops of ethanol were added, and the mixture was then crushed with a pestle in order to convert the powder into a paste. The resulting $\mathrm{CF}_{\mathrm{x}}$ paste was then shaped, placed on a piece of stainless steel mesh, and subsequently pressed into it, yielding a $\mathrm{CF}_{\mathrm{x}}$ cathode.

\subsubsection{Electrolyte Fabrication}

The electrolyte used in the $\mathrm{CF}_{\mathrm{x}}$ batteries was a $1 \mathrm{M}$ solution of lithium hexafluorophosphate $\left(\mathrm{LiPF}_{6}\right)$ salt dissolved in propylene carbonate $(\mathrm{PC})$. To make this electrolyte, all ingredients were inserted into an argon glove box, as seen in Figure 2.4. Once inside, since the molar mass of $\mathrm{LiPF}_{6}$ is quoted by Sigma-Aldrich to be $151.91 \mathrm{~g} / \mathrm{mol}, 3.0382 \mathrm{~g}$ was weighed out. This was then placed into a bottle and $20 \mathrm{~g}$ of PC was then added to it. The bottle was then sealed, shaken, and then left for 48 hours in order to allow the $\mathrm{LiPF}_{6}$ salt to fully dissolve in the PC.

\subsubsection{Battery Assembly}

The process of battery assembly began with all necessary components being gathered and placed in a stainless steel mesh tray. Those components include two coin cell cases, one of which containing a gasket, the $\mathrm{CF}_{\mathrm{x}}$ cathode, an additional piece of stainless steel mesh for the lithium anode, 2 separators, a piece of stainless steel to act 


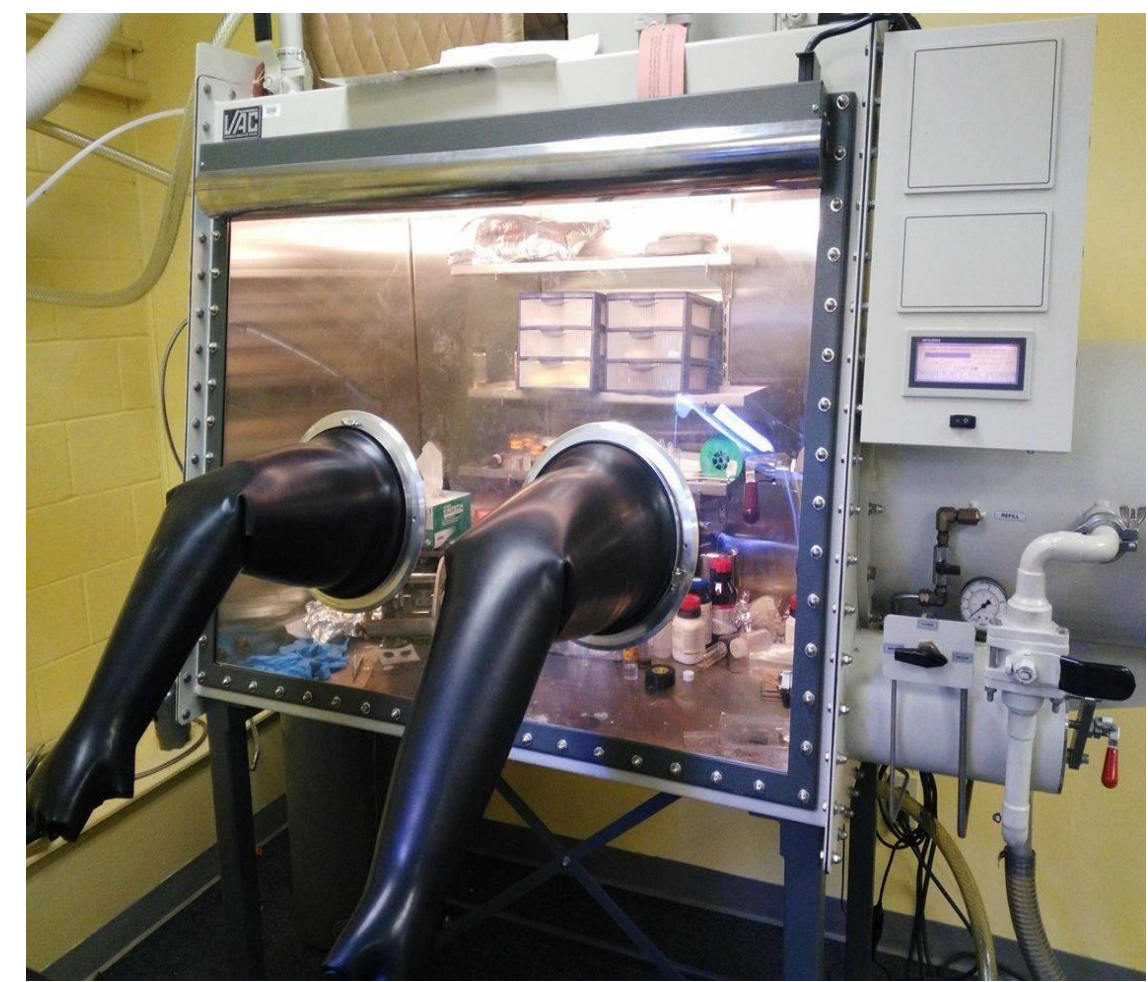

Figure 2.4: Argon glove box used both in the fabrication of the electrolyte as well as in the assembly of the primary batteries.

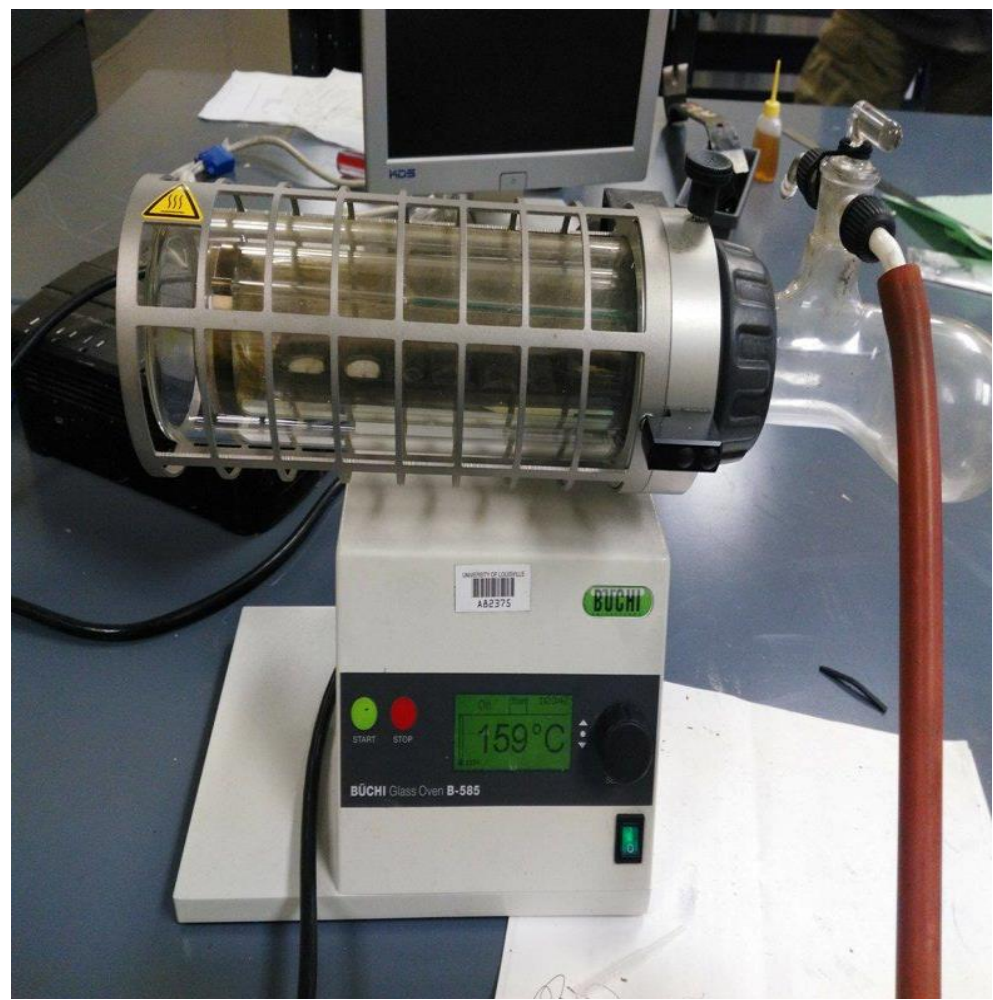

Figure 2.5: Vacuum furnace used to remove moisture from the battery components before they enter the glove box.

as a metal contact, and a stainless steel wave spring to act as a sealing ring. It should 
be noted that the lithium and the electrolyte used in the battery are not included here as they were stored inside the argon glove box. The tray was inserted into the vacuum oven seen in Figure 2.5 after the coin cell case with a gasket was removed from the tray and instead inserted into the compartment to the right of the quartz tube to ensure that the gasket was not damaged by the heat of the furnace. The vacuum furnace was then sealed, and the vacuum pump was started. The furnace was set to $200^{\circ} \mathrm{C}$ and the temperature was held constant at $200^{\circ} \mathrm{C}$ for 2 hours. After this the quartz tube was
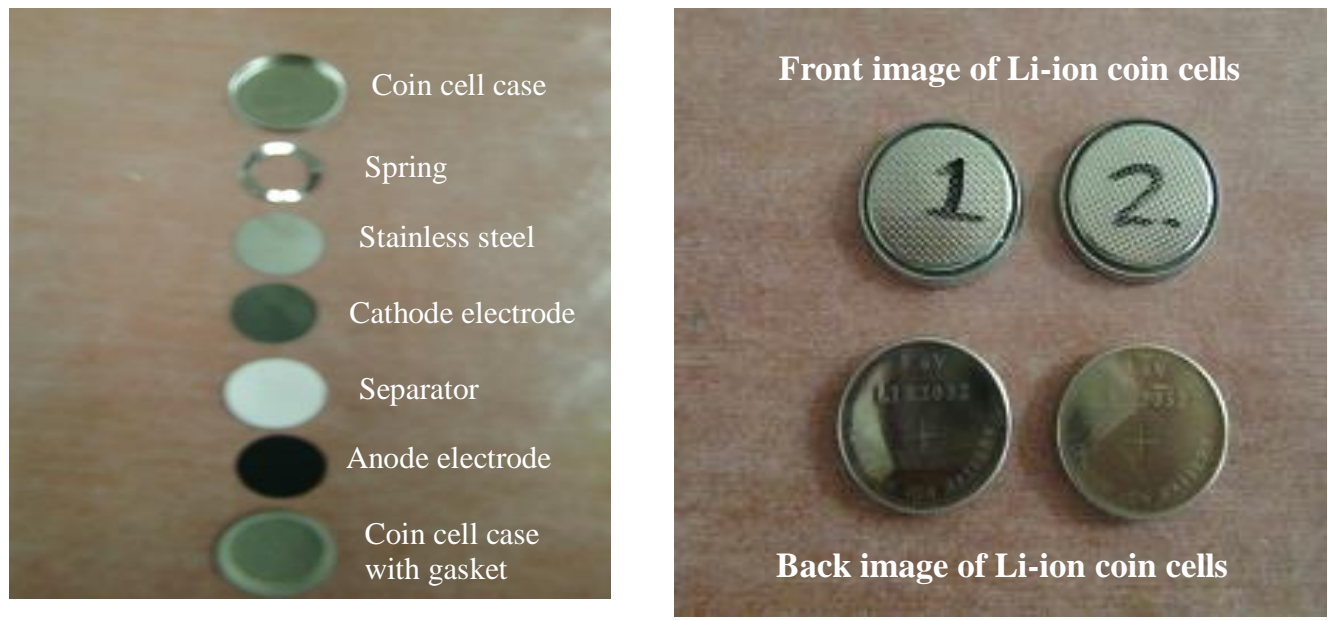

Figure 2.6: Coin cell battery images. (Left) Components in order of assembly from bottom to top. (Right) Completed coin cell batteries.

sealed, and the vacuum pump, as well as the furnace was turned off. The tube, which remained under vacuum was then detached from the furnace and inserted into the glove box. Once the tube was fully inside the glove box, the seal was broken and the components were removed. A piece of lithium metal was pressed into the unused piece of stainless steel mesh using a pair of pliers, making the lithium anode. Then, the lithium anode was place face up in the coin cell case with a gasket. The separators were next placed on top of the anode, and soaked with the LiPF6-PC electrolyte. Next, the $\mathrm{CF}_{\mathrm{x}}$ cathode was placed on top of the separators, face down. The metal contact was added next, followed by the sealing ring. Finally, the other coin cell case was 


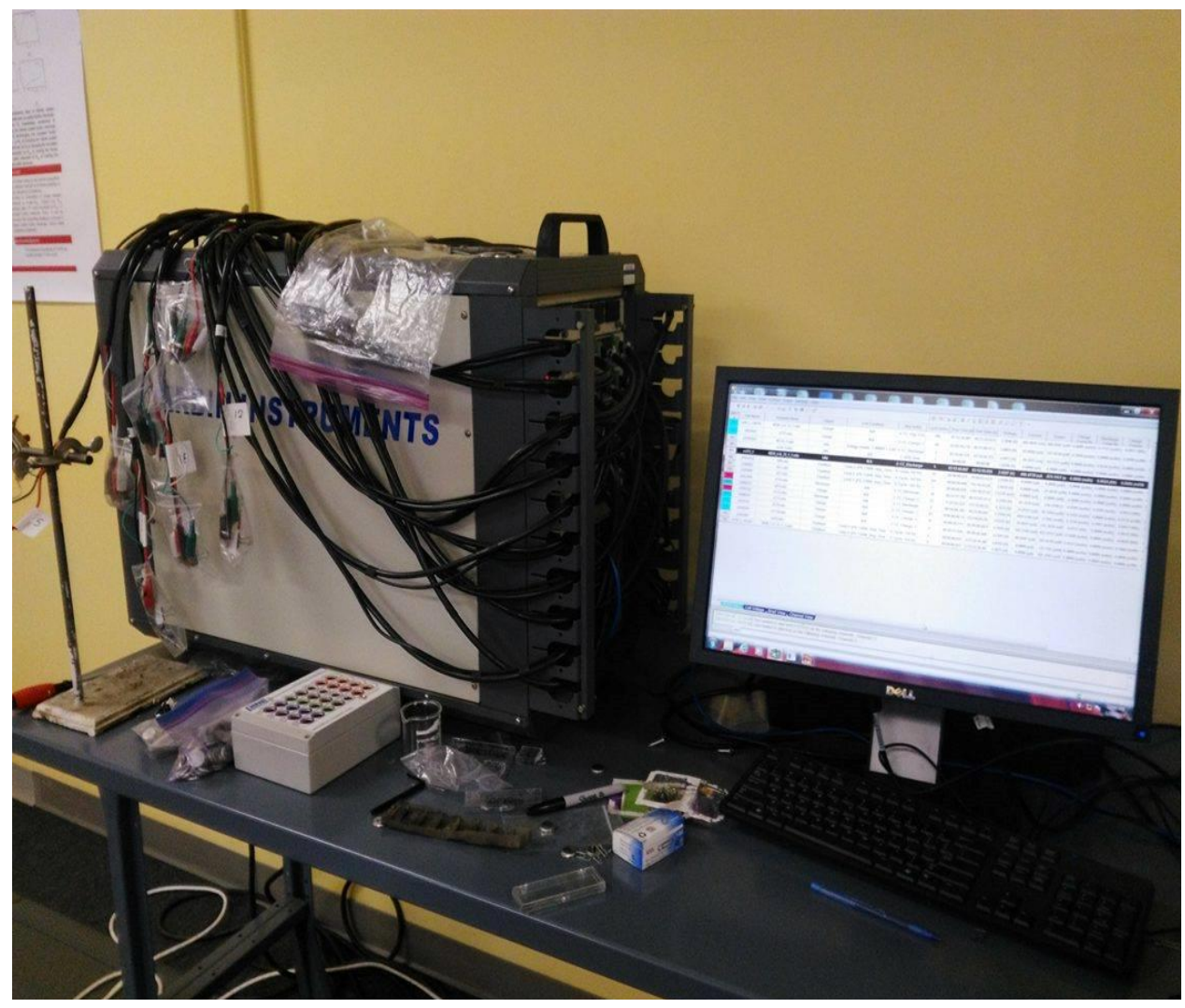

Figure 2.7: Battery tester used to test the discharge capacity of lithium/CF $\mathrm{x}$ primary batteries.

placed on top to complete the battery. After the battery components were assembled, the battery was crimped to ensure proper sealing. The now complete battery was then removed from the glove box.

\subsection{Discharge Capacity Testing}

To test the discharge capacity of the primary lithium/ $\mathrm{CF}_{\mathrm{x}}$ battery, it was attached to an Arbin ${ }^{\mathrm{TM}}$ battery tester as seen in Figure 2.7. The battery started charged to $\sim 3.0 \mathrm{~V}$ and was then allowed to discharge at low current until it reached $1.5 \mathrm{~V}$. The data was recorded and then exported to a spreadsheet for analysis. 


\section{CHAPTER 3: RESULTS AND CONCLUSIONS}

\subsection{Imaging of CNCs}

In order to evaluate to quality of the CNCs resulting from annealing, samples were tested using transition electron microscopy (TEM). Results demonstrate, that annealing generated homogeneous CNCs of radius $3 \mathrm{~nm}$. After the etching, TEM images were again taken, as seen in Figure 3.1. This supports the efficacy of the precursor, the annealing process, as well as the etching in generating CNCs.

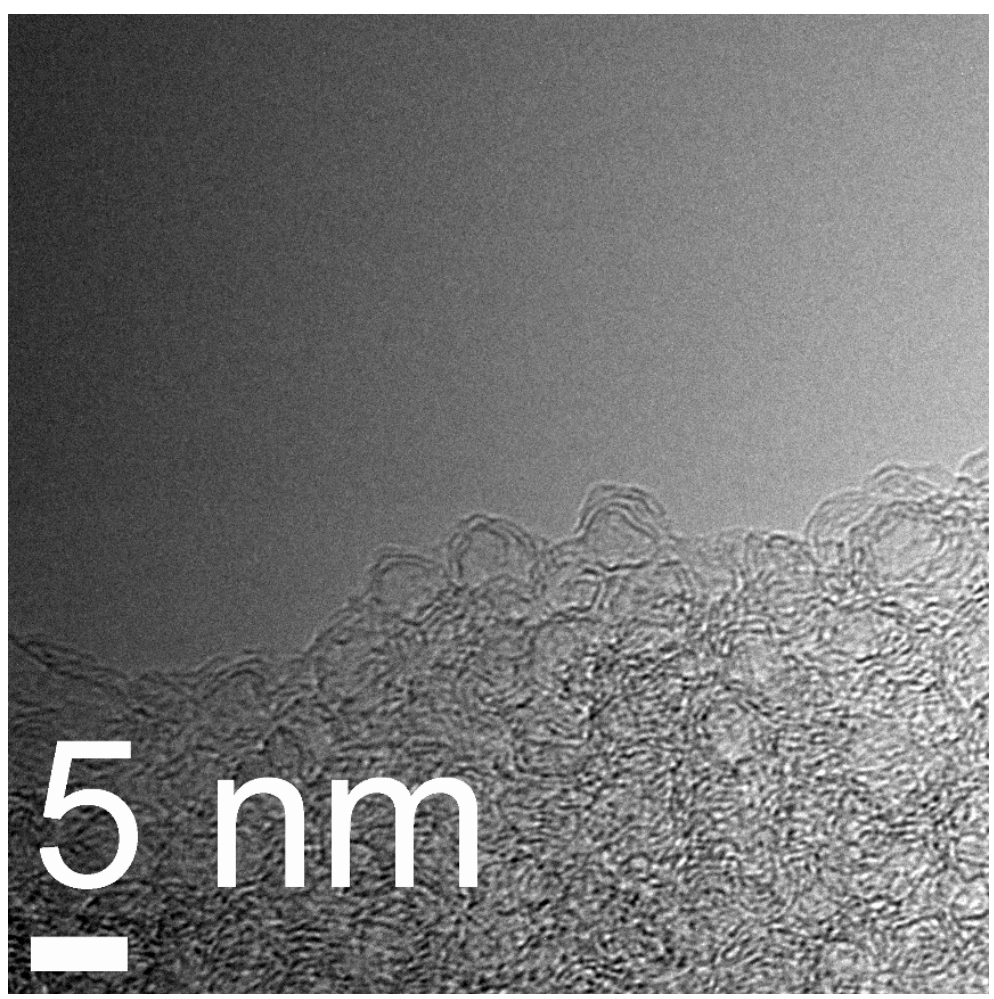

Figure 3.1: TEM image of etched pristine CNCs.

\subsection{Raman and XPS Data for Fluorinated CNCs}

In order to characterize the CNCs after they had undergone fluorination, samples were tested both with Raman spectroscopy and with x-ray photoelectron spectroscopy (XPS). The results of said tests can be found in Figure 3.2. The first 

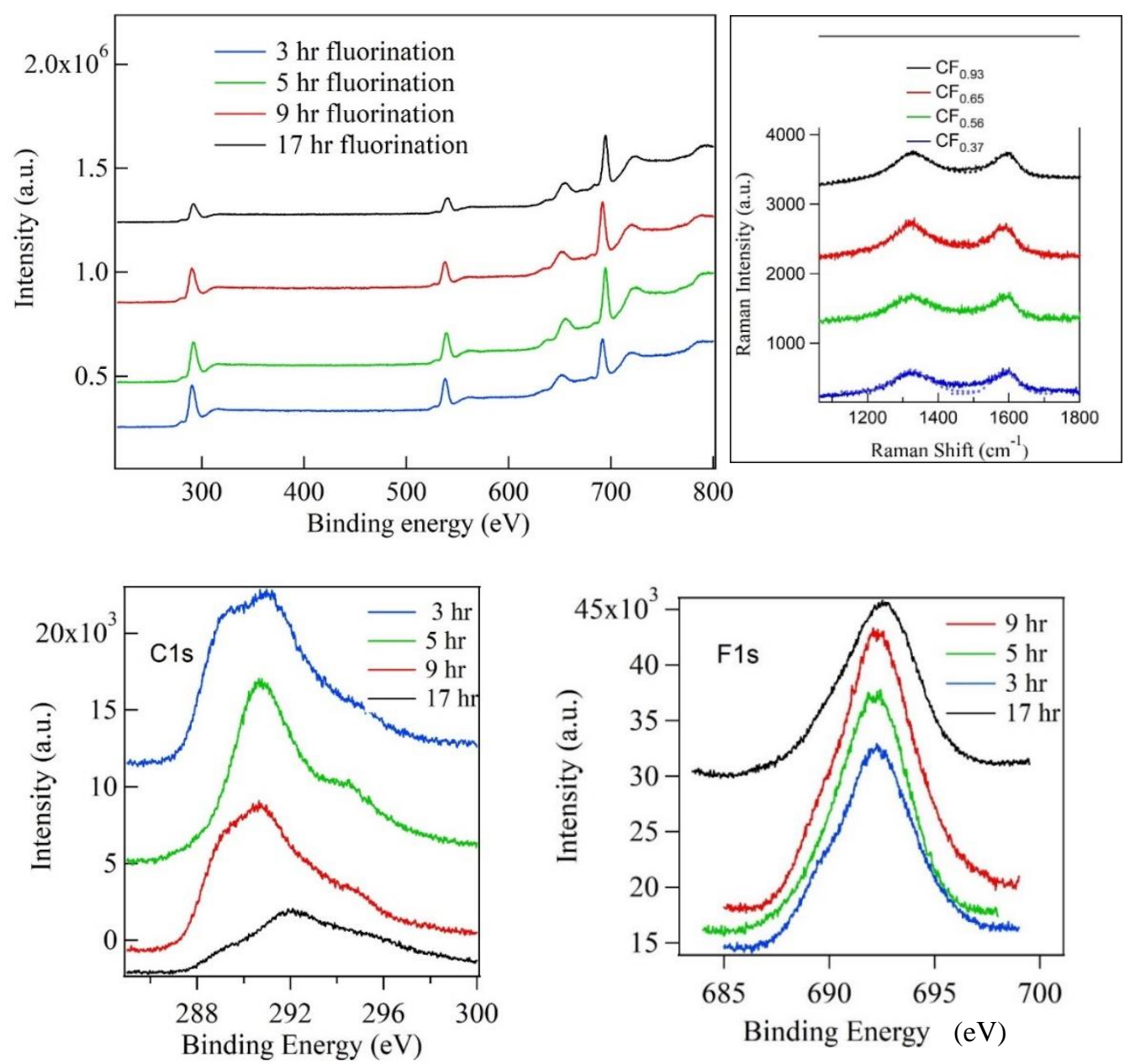

Figure 3.2: XPS and Raman spectroscopy data for CNCs having undergone varying degrees of fluorination. (Upper Left) Full XPS scan. (Upper Right) Ramen Spectroscopy Results. (Lower Left) Detailed view of the C1s peak. (Lower Right) Detailed view of the F1s peak.

point of interest is that when looking at the full XPS scan it becomes evident that for increased fluorination times, the concentration of fluorine in the sample increases as one would expect. This can be further accentuated by examining and comparing the peaks caused by carbon and fluorine in more detail, as can be done by comparing their subsets of the spectrum. By analyzing the areas under these curves, and accounting for the relative sensitivity factors of carbon and fluorine, it becomes possible to calculate the ratio of carbon to fluorine in the sample, which in turn allows for the determining of the $\mathrm{x}$ in $\mathrm{CF}_{\mathrm{x}}$ for the samples. By doing this analysis, it was determined 
that the $\mathrm{x}$ values were $0.37,0.56,0.65$, and 0.93 for the $3,5,9$, and 17 hour fluorinated samples respectively. This means that the discharge capacity measurements for batteries made from these samples should be more than sufficient to be able to determine the efficacy of this method of preparing high capacity lithium/CF $\mathrm{x}$ primary batteries.

\subsection{First Cycle Discharge Capacity Measurements}

With the fluorinated CNCs characterized, it is possible to determine the discharge capacity of the primary batteries made using them. The results for these batteries, as well as the result for a battery made with pristine CNCs instead of fluorinated CNCs to act a point of reference can be seen in Figure 3.3. It immediately

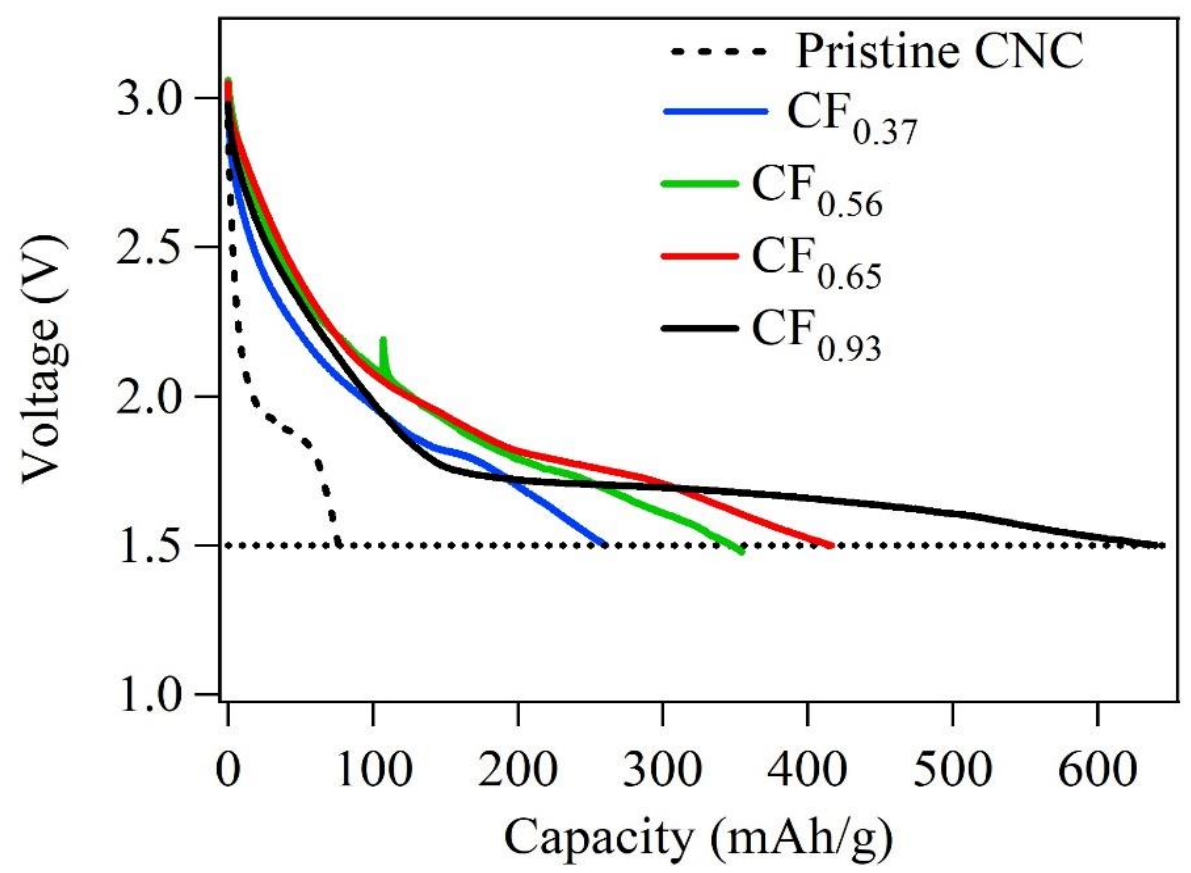

Figure 3.3: Voltage versus first cycle discharge capacity of lithium/ $\mathrm{CF}_{\mathrm{x}}$ primary batteries for varying values of $\mathrm{x}$. Data for pristine CNCs are also plotted for reference.

becomes evident that as the fluorination increases, the discharge capacity of the battery increases. Looking at the numerical values acquired by analyzing the data, the first cycle discharge capacity of the pristine CNCs is just $84 \mathrm{mAh} / \mathrm{g}$. However for the 
fluorinated samples, the specific capacity is found to be $260,355,416$, and finally 660 $\mathrm{mAh} / \mathrm{g}$ for the 3, 5, 9, and 17 hour fluorinated samples respectively. These results imply that the optimal $x$ value has not yet been reached, which in turn means that there is the potential for further increase of the specific capacity.

\subsection{Conclusions}

Based off of the calculated specific capacities, it is clear that by functionalizing CNCs with fluorine good quality primary batteries can be made relatively inexpensively and without any extremely prohibitive step in the production process. Another important detail to note is that over the course of this project, there were no appreciable changes in the quality of the CNCs or their fluorination. This implies that both the initial CNC structure, as well as the fluorine bonding to said structure is quite stable. One potential area for future work would be to optimize the $\mathrm{x}$ value to determine what the maximum achievable value for specific capacity is. Another area of this project which could be improved upon would be the time needed to fluorinate the samples. This could be improved in two different ways in particular. First, experimenting with different methods for cooling the plasma system could potentially alleviate the need to stop fluorination to allow the system to cool, and thus reduce the amount of downtime required. Second, it is potentially possible to modify the plasma system slightly in order to increase the possible power input that the system could use effectively, allowing the fluorination process to occur more quickly. All things considered, the method of fabricating and functionalizing CNCs for use in $\mathrm{CF}_{\mathrm{x}}$ batteries detailed in this thesis is a promising method to yield high capacity primary batteries. 


\section{REFERENCES}

1. Averill, B. and P. Eldredge, Chemistry, principles, patterns, and applications. 2005.

2. Geim, A.K. and K.S. Novoselov, The rise of graphene. Nat Mater, 2007. 6(3): p. 183191.

3. Castro Neto, A.H., et al., The electronic properties of graphene. Reviews of Modern Physics, 2009. 81(1): p. 109-162.

4. Savchenko, A., Materials science. Transforming graphene. Science, 2009. 323(5914): p. 589-90.

5. Wei, D., et al., Synthesis of N-Doped Graphene by Chemical Vapor Deposition and Its Electrical Properties. Nano Letters, 2009. 9(5): p. 1752-1758.

6. Jiao, L., et al., Narrow graphene nanoribbons from carbon nanotubes. Nature, 2009. 458(7240): p. 877-880.

7. Fuchs, J.N. and M.O. Goerbig, Introduction to the physical properties of graphene. 2008.

8. Wallace, P.R., The Band Theory of Graphite. Phys. Rev., 1947. 71(9): p. 622-634.

9. Partoens, B. and F.M. Peeters, From graphene to graphite: Electronic structure around the K point. PHYSICAL REVIEW B, 2006. 74(7): p. 075404.

10. Berger, C., et al., Ultrathin Epitaxial Graphite: 2D Electron Gas Properties and a Route toward Graphene-based Nanoelectronics. The Journal of Physical Chemistry B, 2004. 108(52): p. 19912-19916.

11. Robinson, J., et al., Nucleation of Epitaxial Graphene on SiC(0001). ACS Nano, 2010. 4(1): p. 153-158.

12. Jariwala, D., A. Srivastava, and P.M. Ajayan, Graphene synthesis and band gap opening. J Nanosci Nanotechnol, 2011. 11(8): p. 6621-41.

13. KAWASAKI, T., et al., DOUBLE ATOMIC LAYERS OF GRAPHENE/MONOLAYER $h-B N$ ON Ni(111) STUDIED BY SCANNING TUNNELING MICROSCOPY AND SCANNING TUNNELING SPECTROSCOPY. Surface Review and Letters, 2002. 09(03n04): p. 14591464.

14. Giovannetti, G., et al., Substrate-induced band gap in graphene on hexagonal boron nitride: Ab initio density functional calculations. Phys. Rev. B, 2007. 76(7).

15. Shemelia, P. and S.K. Nayak, Electronic structure and band-gap modulation of graphene via substrate surface chemistry. Applied Physics Letters, 2009. 94(3).

16. $\mathrm{Ci}$, L., et al., Atomic layers of hybridized boron nitride and graphene domains. Nat Mater, 2010. 9(5): p. 430-435.

17. Berger, C., et al., Electronic Confinement and Coherence in Patterned Epitaxial Graphene. Science, 2006. 312(5777): p. 1191-1196.

18. Oku, T., et al., Formation, Atomic Structures and Properties of Carbon Nanocage Materials, in Carbon: The Future Material for Advanced Technology Applications, G. Messina and S. Santangelo, Editors. 2006, Springer Berlin Heidelberg: Berlin, Heidelberg. p. 187-216.

19. Wang, X.X., et al., Carbon nanocages: a new support material for Pt catalyst with remarkably high durability. Sci Rep, 2014. 4: p. 4437.

20. Lyu, Z., et al., Hierarchical carbon nanocages as high-rate anodes for Li- and Na-ion batteries. Nano Research, 2015. 8(11): p. 3535-3543.

21. Ziolkowska, D.A., et al., Simple synthesis of highly uniform bilayer-carbon nanocages. Carbon, 2017. 115: p. 617-624. 
22. Batzill, M., The surface science of graphene: Metal interfaces, CVD synthesis, nanoribbons, chemical modifications, and defects. Surface Science Reports, 2012. 67(3-4): p. 83-115.

23. RAO, C.N.R., et al., GRAPHENE: SYNTHESIS, FUNCTIONALIZATION AND PROPERTIES. International Journal of Modern Physics B, 2011. 25(30): p. 4107-4143.

24. Elias, D.C., et al., Control of Graphene's Properties by Reversible Hydrogenation: Evidence for Graphane. Science, 2009. 323(5914): p. 610-613.

25. Gómez-Navarro, C., et al., Electronic Transport Properties of Individual Chemically Reduced Graphene Oxide Sheets. Nano Letters, 2007. 7(11): p. 3499-3503.

26. Linden, D. and T.B. Reddy, Handbook of Batteries. Third Edition ed. 2002.

27. Greatbatch, W., et al., Lithium/Carbon Monofluoride (Li/CFX): A New Pacemaker Battery. Pacing and Clinical Electrophysiology, 1996. 19(11): p. 1836-1840.

28. Read, J., et al., Performance Evaluation of Commercial CFx Materials in Lithium Batteries. 2009, Army Research Laboratory.

29. Zhang, Q., et al., Progress towards high-power Li/CFx batteries: electrode architectures using carbon nanotubes with CFx. Phys Chem Chem Phys, 2015. 17(35): p. 22504-18. 


\section{CURRICULUM VITA}

NAME: $\quad$ Nathan L. Smith

ADDRESS: Department of Physics

102 Natural Sciences Building

215 Eastern Parkway

Louisville, KY 40208

DOB: $\quad$ July 19, 1993

EDUCATION: B.S. Physics

Georgetown College

2011-2015

M.S Physics

University of Louisville

2015-2017 